\title{
Analytical and Numerical Investigation of Lacing Wire Damage Induced Mistuning in Turbine Blade Packet
}

\author{
Mangesh S. Kotambkar and Animesh Chatterjee \\ Department of Mechanical Engineering, Visvesvaraya National Institute of Technology, Nagpur 440010, India \\ Correspondence should be addressed to Mangesh S. Kotambkar; mskotamb@yahoo.com
}

Received 15 August 2014; Accepted 12 November 2014; Published 9 December 2014

Academic Editor: Abdelkrim Khelif

Copyright (c) 2014 M. S. Kotambkar and A. Chatterjee. This is an open access article distributed under the Creative Commons Attribution License, which permits unrestricted use, distribution, and reproduction in any medium, provided the original work is properly cited.

\begin{abstract}
Investigations of modal parameters for a mistuned packet of turbine blades due to lacing wire damage are reported using analytical and numerical studies with a simplified model. The turbine blade is assumed to be an Euler-Bernoulli beam connected with a lacing wire which is modeled as a mass less linear elastic spring. Thus, the blade is considered as a continuous system and lacing wire as a discrete system. The analytical results using Eigen value analysis are compared with numerical results obtained using commercial finite element package. In real life situation, though not reported in the literature, it is the failure of lacing wire that occurs quite often compared to the turbine blade and acts as precursor to the subsequent blade damage if it goes undetected. Therefore, studying the modal parameters of the grouped turbine blades in the context of lacing wire failure becomes important. The effect of variation of lacing wire location and stiffness indicative of damage resulting in the loss of stiffness on modal parameters is investigated. The study reveals a lot of fundamental understandings pertaining to dynamic behavior of grouped blades compared to the stand-alone blade under the influence of damaged lacing wire.
\end{abstract}

\section{Introduction}

Turbine is the most widely used prime movers in power plants, turbo engines, and compressors in aircrafts and also in auxiliary turbo driven equipment such as turbo pumps. Turbine blade vibration and its failure under high cycle fatigue is an important area of research studies due to its critical applications. The blade failures are mainly due to resonant stresses when one of the natural frequencies of blade-disk system matches the nozzle passing frequency. Design of these turbomachine blades thus critically depends on accurate understanding of the blade vibration characteristics under varied operating conditions. However, modeling and analysis of turbine blade vibration becomes quite complex due to continuously tapered and twisted cross-section and blade to blade dynamic coupling through lacing wire or shroud rings.

Almost $50 \%$ of low pressure steam turbine blade failure is due to fatigue caused by vibration [1]. A turbine blade is a complex geometry having aerofoil shape with varying width and thickness along its length. Most of the early research works have been based on simplified cantilever beam modeling where effects of root flexibility and crack in the stand-alone blade have been studied [2-9]. However, the dynamics of grouped blades is even more complex than a free stand-alone blade.

Rao [10] has summarized the vibration behavior of turbine blades studied numerically and experimentally in the past in his book. In the recent past, research on turbine blade has focused on blades in a packet. There are many advantages of packeted turbine blades connected with lacing wire. For instance, resultant natural frequency increases with the number of blades in group and stiffening due to interconnection of blades with the lacing wire.

Smith [11] has made significant contribution in determining the group frequencies and mode shapes of a blade packet. He also discussed the use of lacing wire in turbine blade groups. Ellington and McCallion [12] simplified Smith's analysis by using finite difference calculus to the special case of blade group with a tie wire which joins the blade tips together.

Prohl [13] used lumped parameter approach by considering series of concentrated masses and concentrated stiffness 
to model the blade group. A modified Holzer technique was used to calculate the natural frequencies and mode shapes. Rao [14] employed energy approach using Hamilton's principle to derive the equations of motion and the boundary conditions.

Bajaj [15] has used the finite element method to determine the natural frequencies of packeted blades in coupled bending-bending-torsion modes. Thomas and Belek [16] have also used finite element model for a group of blades with rectangular cross-section to find modal parameters in the tangential mode. Salama and Petyt [17] have used finite element model and periodical structural analysis of the tangential vibration of packeted blades; both the positions of the lacing wire and rotations were taken into account.

Lim and Yoo [18] have studied modal analysis of multiblade system undergoing rotational motion. It is reported that the increase in rotational speed increases stiffness of the system there by increasing the natural frequencies. They have also reported that increase in coupling stiffness increases the gap between the frequency loci. Lim et al. [19] have also reported the frequency split due to $N$ number of blades in an $\mathrm{Np}$ number of packets as $N / \mathrm{Np}+1$. They have considered tapered cantilever beam and used finite element approach for modal analysis.

These research works mainly focused on tuned blade packet, in which all the blades are considered to be identical to each other. However, mistuning may exist in the form of blade to blade variation in structural and geometrical properties due to manufacturing tolerances, operational wear and tear, or incipient crack growth. Effect of mistuning in a blade group was first investigated by Ewins [20]. Wei and Pierre [21] studied mistuning caused by geometric variations and reported that even a small mistuning in such cases can lead to vibration localization and amplification of stress amplitudes in the blades. Castanier and Pierre [22] have presented a review on reduced-order modeling, simulation, and analysis of the vibration of bladed disks found in gas-turbine engines, in which applications to system identification and design are also considered. An emphasis is placed on key developments that have enabled better prediction and understanding of the forced response of mistuned bladed disks, especially with respect to assessing and mitigating the harmful impact of mistuning on blade vibration, stress increases, and attendant high cycle fatigue.

Hou [23] worked with lumped parameter model, in which the local degradation in stiffness due to blade crack is expressed with a flexibility matrix. Effect of near root blade crack on stability of a packeted blade has been investigated by Huang and Kuang [24]. Fang et al. [25] analysed the mistuned system as cantilever beams interconnected with springs and used fracture mechanics to determine crack induced stiffness loss in a beam (Figure 4). It is reported that crack in the blade may not cause a significant frequency change, but it may lead to forced vibration localization in a periodic structure under loss of cyclic symmetry. Saito et al. [26] considered crack as a nonlinear stiffness and used reduced order modeling for studying the effect of blade damage on vibration response characteristics.
In all these research works, mistuning has been limited to blade damage only. However, practical experience shows that lacing wire damage or breakages are more frequent than blade damage during the operational period of the turbine as they come directly in the path of steam flow/gas flow. The damage may be initially a small surface crack and if left unattended or undetected, such damages finally induce stress localization in the blades leading to blade failure. Unfortunately not much research work has been reported in this area, particularly for blade packets with lacing wire damage. The present work focuses on investigating the effect of lacing wire damage on loss of cyclic symmetry in a blade packet and subsequent characteristic changes in the modal properties. Both lacing wire at the blade tip and lacing wire at an intermediate position are considered. Effect of relative stiffness of the lacing wire to blade stiffness on the modal spectrum for a tuned and mistuned blade packet is investigated. Further studies have been carried out to characterize modal properties for varying damage severity and damage at different locations in the lacing wire loop.

\section{Modeling and Analysis of Tuned Blade Packet}

The turbine blades and disk system is cyclic structure consisting of a number of blade packets. Each packet has multiple blades interconnected with lacing wire or shroud ring. Low pressure turbine stages have long slender blades which undergo high bending deformation caused by natural frequencies in the lower operating range. These blades go through transient resonant condition at nozzle passing frequency [10]. In order to limit the vibratory deformations, these blades of low pressure stages are often stiffened with lacing wire connections. These lacing wires are actually rods of circular cross-sections and are connected with the blades using brazing material. A typical turbine blade disk system with lacing wire interconnection is shown in Figure 1. It can be seen that the blades are arranged in groups called blade packets. Each blade packet consists of a small number of interconnected blades (generally five to seven bladse for low pressure turbine stages).

The packeted blade disk configuration can be considered as a domain composed of identical subdomains that have symmetry with respect to an axis. Analysis of only one of the subdomains represents the key in obtaining major savings in computation time. The subdomain or sector builds up the whole domain by rotating the subdomain by $2 \pi / J$, where $J$ is the number of identical sectors. Thus, dynamic modeling and analysis is done for one blade packet (Figure 2) only with appropriate boundary conditions and this saves considerable amount of computational work and time.

In the present study, it is assumed that failure of the lacing wire would occur gradually through crack growth resulting in a reduction in its stiffness. Stiffness value of the lacing wire is varied as a ratio to the blade stiffness. The objective in the present work is mainly to find answers to following questions. 


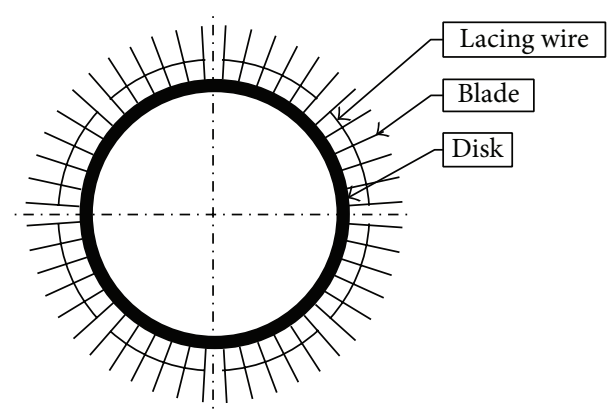

FIGURE 1: Turbine blade disk model with lacing wire.

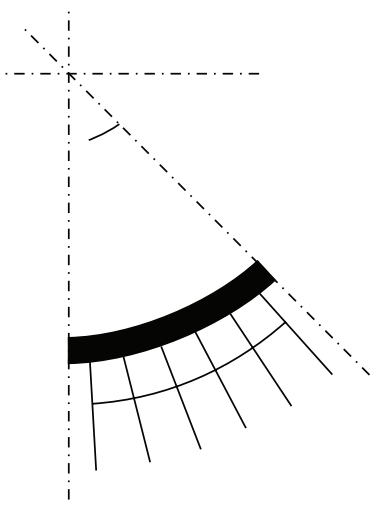

FIGURE 2: One packet of turbine blades with lacing wire.

(i) In what way dynamic behavior and modal characteristics of free standing blade configuration to interconnected blade packet configuration differ and how do these modal characteristics depend on the relative stiffness and location of the lacing wire, number of blades interconnected in a packet, and so forth?

(ii) What would be the qualitative and quantitative shift in the modal properties of the affected blade packet in case of a partial damage/crack or complete breakage in a lacing wire?

(iii) In what way the changes in the modal properties can be used to identify the severity and location of the lacing wire damage?

A mathematical formulation is presented here for a typical three bladed packets with lacing wire positioned at the blade tip initially and subsequently similar analysis for lacing wire at an intermediate position is presented in later sections. Lacing wires are modeled as a spring laterally connected to the blades and governing differential equations of free vibration are derived using Euler-Bernoulli beam theory. The spring force coming from the lacing wire modifies the shear force (SF) boundary condition (BC) at the connection points and this is the fundamental reason why modal properties of an interconnected bladed packet are different from those of free standing blades.

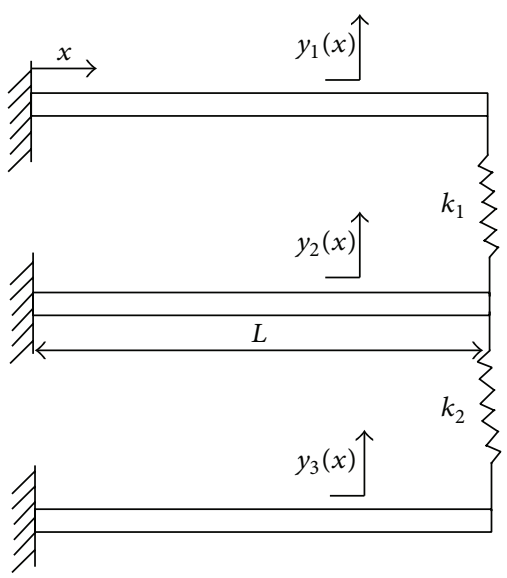

FIGURE 3: Three blades interconnected with lacing wires at free ends.

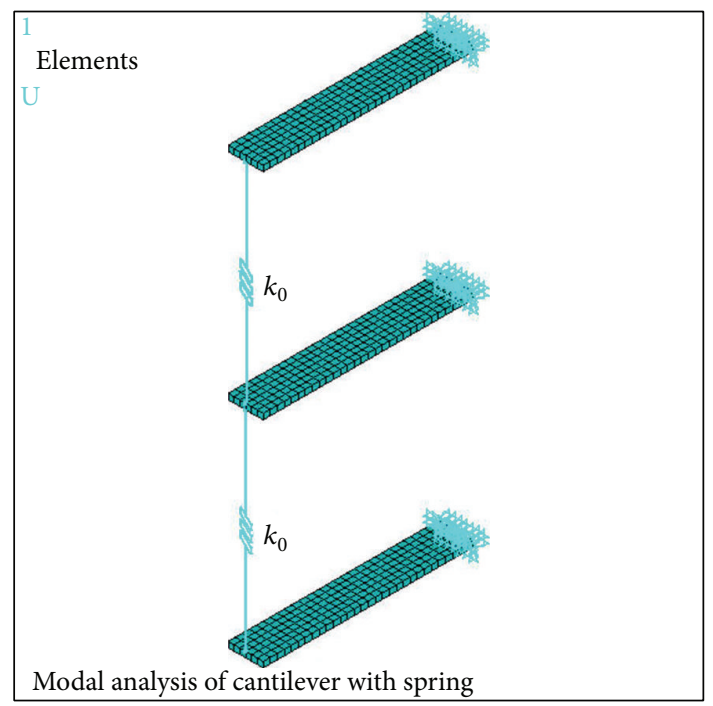

FIGURE 4: Finite element models for three prismatic blades connected with lacing wire at the free end.

\section{Tuned Blade Packet with Lacing Wire Connection at the Blade Tip}

In the analysis, the blades are modeled as parallel cantilever beams fixed at the disk end $(x=0)$ and restrained by the springs (representing the lacing wire stiffening) at the free end $(x=L)$ as shown in Figure 3. Lacing wires have been represented by springs. In tuned condition, the stiffness parameter of the springs $k_{1}$ and $k_{2}$ will be equal. A mistuning caused by a lacing wire crack or damage can be captured in the model by setting different values for the stiffness.

Eigen value analysis for a single blade connected with a lacing wire at the free end is a classical vibration problem found in the standard book [20]. It is extended appropriately for a packet. 
TABLE 1: Natural frequencies $(\beta L)$ of the tuned blade packet with varying stiffness ratios.

\begin{tabular}{cccccc}
\hline Mode & Submode & $\lambda=0.1$ & $\lambda=0.25$ & $\lambda=0.5$ & $\lambda=1.0$ \\
\hline \multirow{3}{*}{1} & I & 1.8751 & 1.8751 & 1.8751 & 1.8751 \\
& II & 1.9189 & 1.9790 & 2.0675 & 2.2135 \\
& III & 1.9978 & 2.1448 & 2.3318 & 2.5932 \\
\hline \multirow{2}{*}{2} & I & 4.6941 & 4.6941 & 4.6941 & 4.6941 \\
& II & 4.6970 & 4.7014 & 4.7087 & 4.7234 \\
& III & 4.7028 & 4.7160 & 4.7383 & 4.7836 \\
\hline \multirow{3}{*}{3} & I & 7.8548 & 7.8548 & 7.8548 & 7.8548 \\
& II & 7.8554 & 7.8563 & 7.8579 & 7.8610 \\
& III & 7.8566 & 7.8594 & 7.8641 & 7.8735 \\
\hline
\end{tabular}

TABLE 2: Comparison of natural frequencies obtained analytically and FEM.

\begin{tabular}{ccccc}
\hline Mode Submode & $\begin{array}{c}\text { Frequency in } \mathrm{Hz} \\
\text { (analytical) }\end{array}$ & $\begin{array}{c}\text { Frequency in } \mathrm{Hz} \\
\text { (FEM) }\end{array}$ & \% error \\
\hline \multirow{3}{*}{1} & I & 72.52 & 73.15 & 0.87 \\
& II & 75.94 & 76.56 & 0.80 \\
& III & 82.32 & 82.90 & 0.71 \\
\hline \multirow{2}{*}{2} & I & 454.45 & 458.04 & 0.78 \\
& II & 455.00 & 458.60 & 0.78 \\
& III & 456.14 & 459.73 & 0.78 \\
\hline & I & 1272.45 & 1284.90 & 0.97 \\
& II & 1272.63 & 1285.10 & 0.97 \\
& III & 1273.06 & 1285.50 & 0.97 \\
\hline
\end{tabular}

The \% error in the results of natural frequencies in the submodes of three bending modes of vibration obtained with both methods is less than $1 \%$.

The equations of motion of free vibration for the blades are given by

$$
\text { EI } \frac{\partial^{4} y_{i}}{\partial x^{4}}+\rho A \frac{\partial^{2} y_{i}}{\partial t^{2}}=0, \quad \text { where } i=1,2,3
$$

The above equation is well known as Euler-Bernoulli equation for a uniform cross-section beam, for which the solution exists in the form

$$
y(x, t)=Y_{i}(x) \cdot \eta(t)
$$

where the mode shape function $Y(x)$ is expressed as

$$
Y(x)=C_{1} \cosh \beta x+C_{2} \sinh \beta x+C_{3} \cos \beta x+C_{4} \sin \beta x .
$$

With

$$
\beta=\left(\omega^{2} \frac{\rho A}{\mathrm{EI}}\right)^{1 / 4}
$$

such that natural frequency can be obtained as

$$
\omega=\beta^{2} \sqrt{\frac{\mathrm{EI}}{\rho A}} .
$$

BC's for the upper blade are as follows.

$$
\text { At } x_{1}=0, y_{1}\left(x_{1}\right)=0 \text { and } y_{1}^{\prime}\left(x_{1}\right)=0 \text {; therefore, } C_{3}=
$$
$-C_{1}$ and $C_{4}=-C_{2}$.

At $x_{1}=l, y_{1}^{\prime \prime}\left(x_{1}\right)=0$, and $y_{1}^{\prime \prime \prime}\left(x_{1}\right)=k_{1}\left(y_{1}-y_{2}\right)$ give two equations BM and SF, respectively,

$C_{1}(\cosh \beta l+\cos \beta l)+C_{2}(\sinh \beta l+\sin \beta l)=0$,

$$
\begin{aligned}
& C_{1}\left[(\sinh \beta l-\sin \beta l)-(\cosh \beta l-\cos \beta l) \frac{k_{1}}{(\beta L)^{3}\left(\mathrm{EI} / L^{3}\right)}\right] \\
& +C_{2}[(\cosh \beta l+\cos \beta l) \\
& \left.\quad-(\sinh \beta l-\sin \beta l) \frac{k_{1}}{(\beta L)^{3}\left(\mathrm{EI} / L^{3}\right)}\right] \\
& +C_{5}(\cosh \beta l-\cos \beta l) \frac{k_{1}}{(\beta L)^{3}\left(\mathrm{EI} / L^{3}\right)} \\
& +C_{6}(\sinh \beta l-\sin \beta l) \frac{k_{1}}{(\beta L)^{3}\left(\mathrm{EI} / L^{3}\right)}=0 .
\end{aligned}
$$

BC's for the middle blade are as follows.

At $x_{2}=0, y_{2}\left(x_{2}\right)=0$ and $y_{2}^{\prime}\left(x_{2}\right)=0$; therefore, $C_{7}=$ $-C_{5}$ and $C_{8}=-C_{6}$.

At $x_{2}=l, y_{2}^{\prime \prime}\left(x_{2}\right)=0$ and $y_{2}^{\prime \prime \prime}\left(x_{2}\right)=k_{2}\left(y_{2}-y_{3}\right)+$ $k_{1}\left(y_{2}-y_{1}\right)$ give two equations BM and SF, respectively,

$C_{5}(\cosh \beta l+\cos \beta l)+C_{6}(\sinh \beta l+\sin \beta l)=0$,

$C_{1}(\cosh \beta l-\cos \beta l) \frac{k_{1}}{(\beta L)^{3}\left(\mathrm{EI} / L^{3}\right)}$

$$
\begin{aligned}
& +C_{2}(\sinh \beta l-\sin \beta l) \frac{k_{1}}{(\beta L)^{3}\left(\mathrm{EI} / L^{3}\right)} \\
& +C_{5}[(\sinh \beta l-\sin \beta l)
\end{aligned}
$$

$$
\left.-(\cosh \beta l-\cos \beta l) \frac{k_{1}+k_{2}}{(\beta L)^{3}\left(\mathrm{EI} / L^{3}\right)}\right]
$$

$$
+C_{6}[(\cosh \beta l+\cos \beta l)
$$

$$
\left.-(\sinh \beta l-\sin \beta l) \frac{k_{1}+k_{2}}{(\beta L)^{3}\left(\mathrm{EI} / L^{3}\right)}\right]
$$




$$
\begin{aligned}
& +C_{9}(\cosh \beta l-\cos \beta l) \frac{k_{2}}{(\beta L)^{3}\left(\mathrm{EI} / L^{3}\right)} \\
& +C_{10}(\sinh \beta l-\sin \beta l) \frac{k_{2}}{(\beta L)^{3}\left(\mathrm{EI} / L^{3}\right)}=0 .
\end{aligned}
$$

BC's for the lower blade are as follows.

$$
\begin{aligned}
& \text { At } x_{3}=0, y_{3}\left(x_{3}\right)=0 \text { and } y_{3}^{\prime}\left(x_{3}\right)=0 \text {; therefore, } C_{11}= \\
& -C_{9} \text { and } C_{12}=-C_{10} . \\
& \text { At } x_{3}=l, y_{3}^{\prime \prime}\left(x_{3}\right)=0 \text { and } y_{3}^{\prime \prime \prime}\left(x_{3}\right)=k_{2}\left(y_{3}-y_{2}\right)_{1} \\
& C_{9}(\cosh \beta l+\cos \beta l)+C_{10}(\sinh \beta l+\sin \beta l)=0, \\
& C_{5}(\cosh \beta l-\cos \beta l) \frac{k_{2}}{(\beta L)^{3}\left(\mathrm{EI} / L^{3}\right)}
\end{aligned}
$$

$$
\mid \begin{array}{ccc}
P & Q & 0 \\
S-\frac{\lambda_{1} R}{(\beta L)^{3}} & P-\frac{\lambda_{1} S}{(\beta L)^{3}} & \frac{\lambda_{1} R}{(\beta L)^{3}} \\
0 & 0 & P \\
\frac{\lambda_{1} R}{(\beta L)^{3}} & \frac{\lambda_{1} S}{(\beta L)^{3}} & S-\frac{\left(\lambda_{1}+\lambda_{2}\right) R}{(\beta L)^{3}} \\
0 & 0 & 0 \\
0 & 0 & \frac{\lambda_{2} R}{(\beta L)^{3}}
\end{array}
$$

where $P=\cosh \beta L+\cos \beta L, Q=\sinh \beta L+\sin \beta L, R=$ $\cosh \beta L-\cos \beta L, S=\sinh \beta L-\sin \beta L$, and $\lambda_{i}=k_{i} /\left(\mathrm{EI} / L^{3}\right)$. Since EI $/ L^{3}$ is a measure of flexural stiffness of a beam, the parameter represents the stiffness ratio between the lacing wire and the cantilever beam.

Although, in the present analytical work, we have considered three blades, the same procedures can be extended for a blade packet having more numbers of blades. The determinant in (11) is a function of two primary variables, $\beta L$ and $\lambda_{i}$. Since all the lacing wire segments are identical in undamaged state, equating the determinant to zero in (11) provides the set of natural frequencies for a tuned blade packet for a given value of stiffness ratio, $\lambda_{i}$. Table 1 presents the natural frequency values of the blade packet for a range of stiffness ratios $(\lambda=0.1,0.25,0.5$, and 1.0$)$.

The numerical approach using finite element analysis (FEA) is carried out with the commercial software ANSYS 13.0. The solid 45 brick element is used for meshing the blade and combin14 element is used for lacing wire. The mesh sensitivity analysis is done to ensure the optimum size of element. The results are compared for $\lambda=0.1$ in Table 2 .

$$
\begin{aligned}
+C_{6}( & \sinh \beta l-\sin \beta l) \frac{k_{2}}{(\beta L)^{3}\left(\mathrm{EI} / L^{3}\right)} \\
+C_{9} & {[(\sinh \beta l-\sin \beta l)} \\
& \left.-(\cosh \beta l-\cos \beta l) \frac{k_{2}}{(\beta L)^{3}\left(\mathrm{EI} / L^{3}\right)}\right]
\end{aligned}
$$

$$
\begin{aligned}
+C_{10} & {[(\cosh \beta l+\cos \beta l)} \\
& \left.-(\sinh \beta l-\sin \beta l) \frac{k_{2}}{(\beta L)^{3}\left(\mathrm{EI} / L^{3}\right)}\right]=0 .
\end{aligned}
$$

Using the above equations, one gets the frequency equation in a determinant form as

$\begin{array}{ccc}0 & 0 & 0 \\ \frac{\lambda_{1} S}{(\beta L)^{3}} & 0 & 0 \\ Q & 0 & 0 \\ \frac{\left(\lambda_{1}+\lambda_{2}\right) S}{(\beta L)^{3}} & \frac{\lambda_{2} R}{(\beta L)^{3}} & \frac{\lambda_{2} S}{(\beta L)^{3}} \\ \frac{\lambda_{2} S}{(\beta L)^{3}} & S-\frac{\lambda_{2} R}{(\beta L)^{3}} & P-\frac{\lambda_{2} S}{(\beta L)^{3}}\end{array} \mid=0$,

The \% error in the results of natural frequencies in the submodes of three bending modes of vibration obtained with both methods is less than $1 \%$.

Corresponding mode shapes (for $\lambda=0.1$ ) for the three submodes in case of first, second, and third modes are presented in Figures 5, 6, and 7.

In Figure 5(a), all the blades are vibrating in phase, which means springs are not undergoing any deformation and thus are not contributing any restraints on the beams. This is why the first submode natural frequency is the same as the natural frequency of the free standing blades.

In Figure 5(b), outer blades are out of phase, whereas the central blade remains undeformed. In Figure 5(c), outer blades are in phase and the central blade is out of phase. This gives higher frequency values for submodes II and III.

The following characteristics can be noted from the frequency values as given in Table 1 .

(i) When blades are grouped into a blade packet, each mode of the free standing blade gets split in several (three in this case) submodes. The first submode natural frequency is the same as the first mode natural 


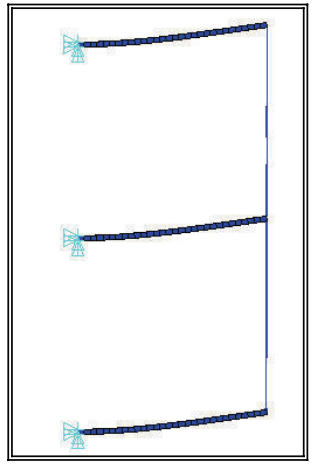

(a) Submode I

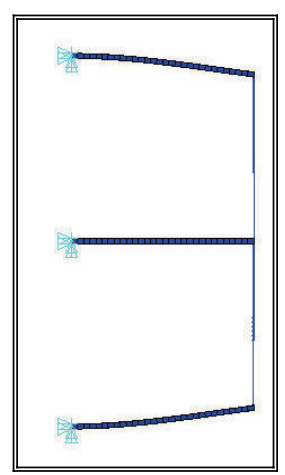

(b) Submode II

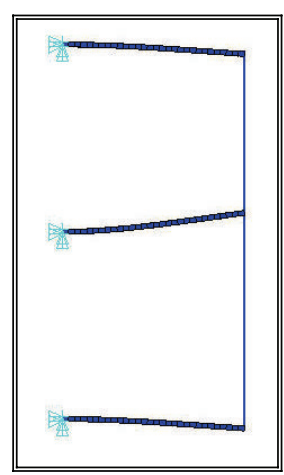

(c) Submode III

Figure 5: Submodes of 1st mode shape.

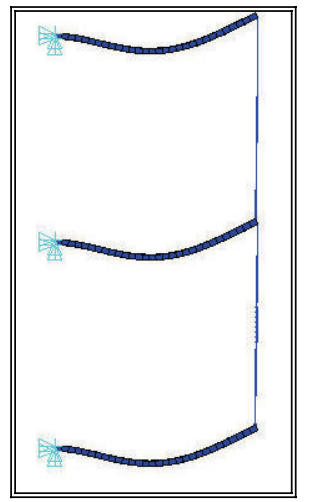

(a) Submode I

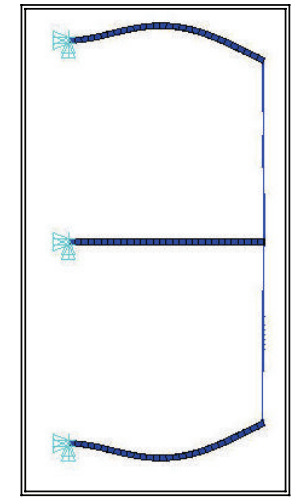

(b) Submode II

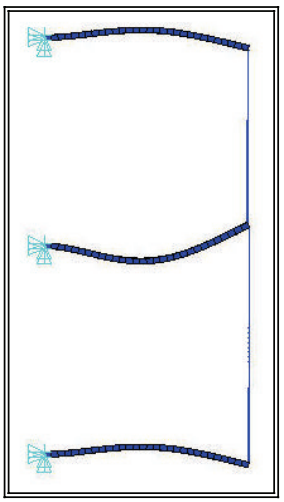

(c) Submode III

FIgURE 6: Submodes of 2nd mode shape.

frequency of the stand-alone blade and the remaining submode frequencies are additional in the frequency spectrum.

(ii) The second and third submode natural frequencies are higher than the basic mode natural frequency and their difference from the basic mode natural frequency is directly related to the stiffness ratio $\lambda$. As $\lambda$ increases, these submode frequencies increase higher and higher (Figure 8). Thus, the values of the second and higher submodes give an indication of the relative strength of the lacing wire stiffness with respect to that of the blade and hence any change in lacing wire stiffness due to a crack or damage is expected to change these frequencies.

(iii) The submode natural frequencies of the first mode are more sensitive to the presence of lacing wire than the frequencies in higher modes. This is due to the position of lacing wire at the blade tip, which happens to be the antinode of first mode shape. Thus, a spring at the blade tip constrains the first mode to maximum extent and thereby changes the frequencies associated with the first mode. Figure 8 shows the same quite distinctly.

\section{Analysis of Mistuned Blade Packet due to Damage in Lacing Wire}

During operation, lacing wire often develops crack resulting in stiffness loss. When the lacing wire undergoes full cracking, the spring stiffness becomes zero. This reduction in stiffness in one of the lacing wire segments introduces mistuning in the blade packet. This modifies the natural frequencies of the blade packet which is investigated and presented below.

Figure 9 shows partially damaged lacing wire segment between second and third blades, whereas the lacing wire segment between first and second blades is presumed to be undamaged. As a result of damage, spring stiffness $k_{2}$ becomes less than $k_{1}$. In case of complete breakage of this lacing wire, $k_{2}$ reduces to zero (Figure 10). A damage severity factor $\alpha$ can be defined as

$$
\alpha=\frac{\Delta k}{k_{1}}, \quad \text { where } \Delta k=k_{1}-k_{2} .
$$

As lacing wire damage increases, $k_{2}$ decreases compared to $k_{1}$ due to which $\Delta k$ goes on increasing and thus damage severity factor $\alpha$ increases from zero to finally one, when the lacing wire undergoes full cracking. 


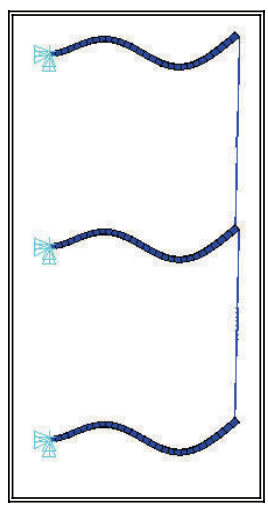

(a) Submode I

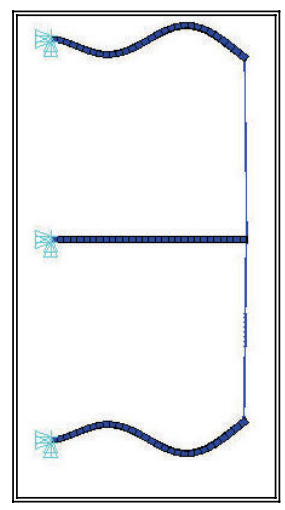

(b) Submode II

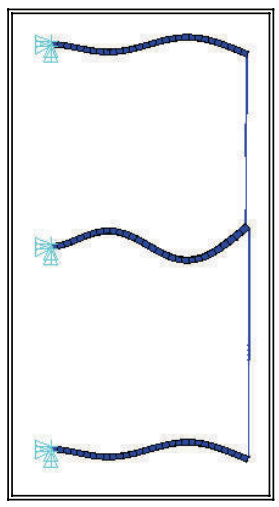

(c) Submode III

Figure 7: Submodes of 3rd mode shape.

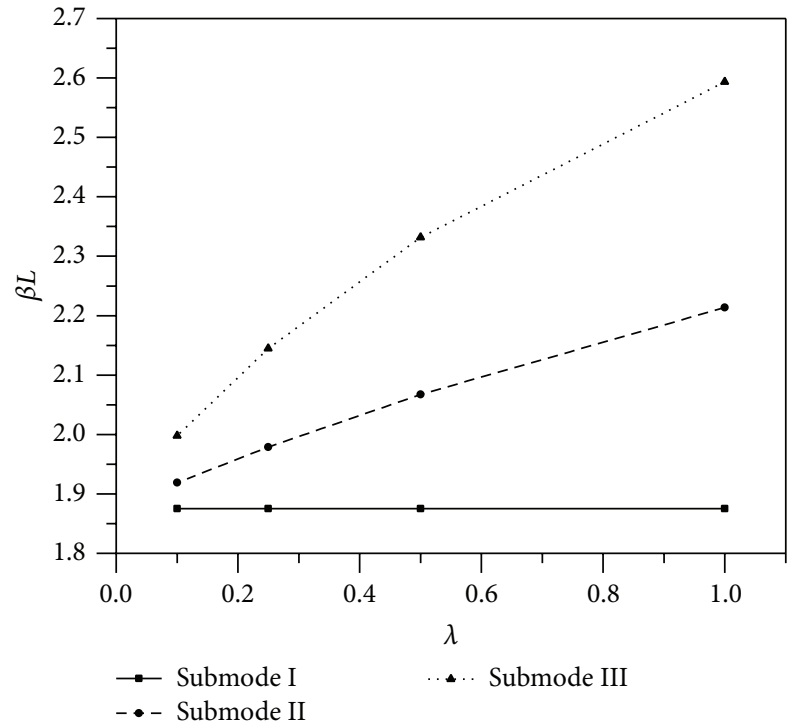

FIGURE 8: Effect of varying stiffness ratios on natural frequencies of submodes of the fundamental mode.

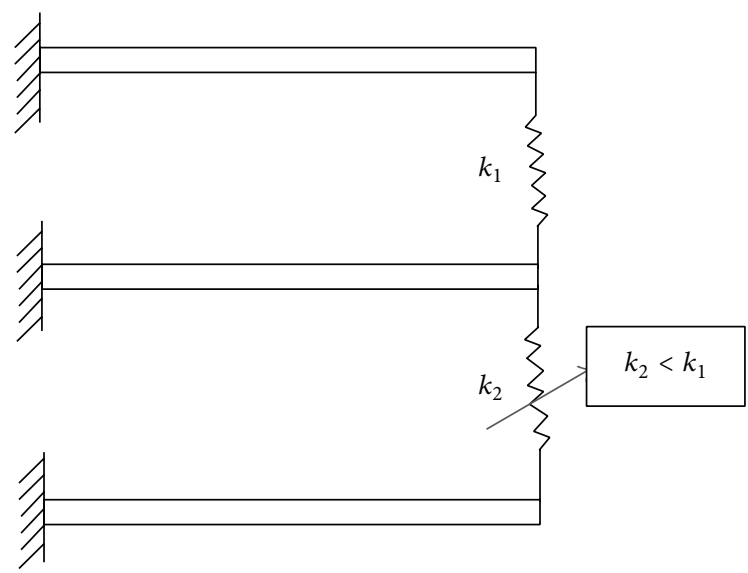

FIGURE 9: Three blades with one partially damaged lacing wire.

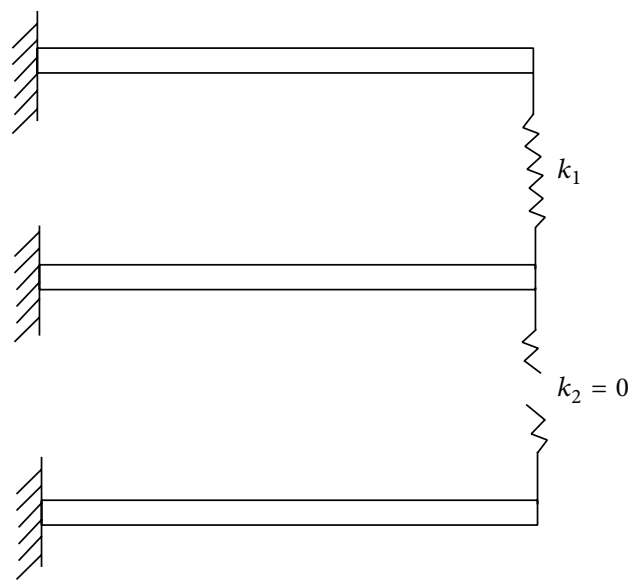

FIGURE 10: Three blades with fully damaged/broken lacing wire at free ends.

For a typical lacing wire damage, frequency determinant equation (11) is evaluated with reduced spring stiffness ratio of $\lambda_{2}$ corresponding to the reduced value of $k_{2}=(1-\alpha) * k_{1}$. The corresponding frequency values of the modified mistuned system for varying degrees of damage severity ( $\alpha$ varying between zero and one) are presented in Tables 3, 4, 5, and 6 for $\lambda=0.1,0.25,0.5$, and 1.0 .

It can be noted from Tables 3, 4, 5, and 6 that all the submode frequencies in all the modes are reducing with the increase in lacing wire damage severity $\alpha$ from 0 to 1 . The drop in submode frequencies is more in the tuned system with the higher value of $\lambda$. It can be further observed that drop in submodes frequency of the fundamental mode is higher compared to submodes frequency of the higher modes.

Therefore, effect of lacing wire damage on mistuning has been shown in Figures 11(a)-11(d) for the submodes of the fundamental mode only for tuned systems $\lambda=0.1,0.25,0.5$, and 1.0. 
TABLE 3: Effect of mistuning due to lacing wire damage (for $\lambda=0.1$ ) on natural frequencies.

\begin{tabular}{lllllllll}
\hline \multirow{2}{*}{ Mode } & \multirow{2}{*}{ Submode } & \multicolumn{6}{c}{$\beta L$ values } \\
& & $\alpha=0$ & $\alpha=0.2$ & $\alpha=0.4$ & $\alpha=0.6$ & $\alpha=0.8$ & $\alpha=1.0$ \\
\hline \multirow{3}{*}{1} & I & 1.8751 & 1.8751 & 1.8751 & 1.8751 & 1.8751 & 1.8751 \\
& II & 1.9189 & 1.9140 & 1.9073 & 1.8987 & 1.8879 & 1.8751 \\
& III & 1.9978 & 1.9872 & 1.9780 & 1.9703 & 1.9642 & 1.9597 \\
\hline \multirow{3}{*}{2} & I & 4.6941 & 4.6941 & 4.6941 & 4.6941 & 4.6941 & 4.6941 \\
& II & 4.6970 & 4.6967 & 4.6962 & 4.6956 & 4.6949 & 4.6941 \\
& III & 4.7028 & 4.7020 & 4.7013 & 4.7007 & 4.7002 & 4.6999 \\
\hline & I & 7.8548 & 7.8548 & 7.8548 & 7.8548 & 7.8548 & 7.8548 \\
3 & II & 7.8554 & 7.8553 & 7.8552 & 7.8551 & 7.8549 & 7.8548 \\
& III & 7.8566 & 7.8564 & 7.8563 & 7.8562 & 7.8561 & 7.8560 \\
\hline
\end{tabular}

TABLE 4: Effect of mistuning due to lacing wire damage (for $\lambda=0.25$ ) on natural frequencies.

\begin{tabular}{lllllllll}
\hline \multirow{2}{*}{ Mode Submode } & \multicolumn{7}{c}{$\beta L$ values } \\
& & $\alpha=0$ & $\alpha=0.2$ & $\alpha=0.4$ & $\alpha=0.6$ & $\alpha=0.8$ & $\alpha=1.0$ \\
\hline \multirow{3}{*}{1} & I & 1.8751 & 1.8751 & 1.8751 & 1.8751 & 1.8751 & 1.8751 \\
& II & 1.9790 & 1.9678 & 1.9526 & 1.9323 & 1.9065 & 1.8751 \\
& III & 2.1448 & 2.1239 & 2.1052 & 2.0895 & 2.0771 & 2.0675 \\
\hline \multirow{3}{*}{2} & I & 4.6941 & 4.6941 & 4.6941 & 4.6941 & 4.6941 & 4.6941 \\
& II & 4.7014 & 4.7005 & 4.6994 & 4.6979 & 4.6962 & 4.6941 \\
& III & 4.7160 & 4.7139 & 4.7121 & 4.7107 & 4.7095 & 4.7087 \\
\hline & I & 7.8548 & 7.8548 & 7.8548 & 7.8548 & 7.8548 & 7.8548 \\
3 & II & 7.8563 & 7.8561 & 7.8559 & 7.8556 & 7.8552 & 7.8548 \\
& III & 7.8594 & 7.8590 & 7.8586 & 7.8583 & 7.8580 & 7.8579 \\
\hline
\end{tabular}

TABLE 5: Effect of mistuning due to lacing wire damage (for $\lambda=0.5$ ) on natural frequencies.

\begin{tabular}{llllllll}
\hline \multirow{2}{*}{ Mode Submode } & \multicolumn{7}{c}{$\beta L$ values } \\
& & $\alpha=0$ & $\alpha=0.2$ & $\alpha=0.4$ & $\alpha=0.6$ & $\alpha=0.8$ & $\alpha=1.0$ \\
\hline \multirow{3}{*}{1} & I & 1.8751 & 1.8751 & 1.8751 & 1.8751 & 1.8751 & 1.8751 \\
& II & 2.0675 & 2.0480 & 2.0211 & 1.9844 & 1.9363 & 1.8751 \\
& III & 2.3318 & 2.3005 & 2.2721 & 2.2479 & 2.2285 & 2.2135 \\
\hline \multirow{3}{*}{2} & I & 4.6941 & 4.6941 & 4.6941 & 4.6941 & 4.6941 & 4.6941 \\
2 & II & 4.7087 & 4.7070 & 4.7047 & 4.7018 & 4.6982 & 4.6941 \\
& III & 4.7383 & 4.7340 & 4.7304 & 4.7274 & 4.7251 & 4.7234 \\
\hline & I & 7.8548 & 7.8548 & 7.8548 & 7.8548 & 7.8548 & 7.8548 \\
3 & II & 7.8579 & 7.8575 & 7.8570 & 7.8564 & 7.8556 & 7.8548 \\
& III & 7.8641 & 7.8632 & 7.8624 & 7.8618 & 7.8613 & 7.8610 \\
\hline
\end{tabular}

From Tables 3, 4, 5, and 6 and corresponding Figures 11(a)-11(d), it can be noted that, with a damage in a lacing wire, the first submode frequency is invariant, but other submode frequencies vary and in fact they decrease when compared to a blade packet with no damage in lacing wire. The change in natural frequencies is augmented with stiffer connection (i.e., higher $\lambda$ ) and is more prominent in fundamental mode compared to higher modes.
TABLE 6: Effect of mistuning due to lacing wire damage (for $\lambda=1$ ) on natural frequencies.

\begin{tabular}{cccccccc}
\hline \multirow{2}{*}{ Mode Submode } & \multicolumn{7}{c}{$\beta L$ values } \\
& & $\alpha=0$ & $\alpha=0.2$ & $\alpha=0.4$ & $\alpha=0.6$ & $\alpha=0.8$ & $\alpha=1.0$ \\
\hline \multirow{3}{*}{1} & I & 1.8751 & 1.8751 & 1.8751 & 1.8751 & 1.8751 & 1.8751 \\
& II & 2.2135 & 2.1824 & 2.1384 & 2.0768 & 1.9917 & 1.8751 \\
& III & 2.5932 & 2.5514 & 2.5129 & 2.4797 & 2.4526 & 2.4314 \\
\hline & I & 4.6941 & 4.6941 & 4.6941 & 4.6941 & 4.6941 & 4.6941 \\
2 & II & 4.7234 & 4.7200 & 4.7154 & 4.7095 & 4.7023 & 4.6941 \\
& III & 4.7836 & 4.7749 & 4.7675 & 4.7615 & 4.7568 & 4.7533 \\
\hline & I & 7.8548 & 7.8548 & 7.8548 & 7.8548 & 7.8548 & 7.8548 \\
3 & II & 7.8610 & 7.8603 & 7.8593 & 7.8580 & 7.8565 & 7.8548 \\
& III & 7.8735 & 7.8718 & 7.8702 & 7.8689 & 7.8680 & 7.8548 \\
\hline
\end{tabular}

TABLE 7: Natural frequencies ( $\beta L$ values) of the tuned blade packet with varying stiffness ratios (lacing wire at $2 / 3 \mathrm{rd}$ of blade span).

\begin{tabular}{cccccc}
\hline Mode & Submode & $\lambda=0.1$ & $\lambda=0.25$ & $\lambda=0.5$ & $\lambda=1.0$ \\
\hline \multirow{3}{*}{1} & I & 1.8751 & 1.8751 & 1.8751 & 1.8751 \\
& II & 1.8886 & 1.9082 & 1.9396 & 1.9978 \\
& III & 1.9146 & 1.9694 & 2.0511 & 2.1884 \\
\hline \multirow{3}{*}{2} & I & 4.6941 & 4.6941 & 4.6941 & 4.6941 \\
& II & 4.6946 & 4.6954 & 4.6967 & 4.6993 \\
& III & 4.6957 & 4.6980 & 4.7019 & 4.7065 \\
\hline \multirow{3}{*}{3} & I & 7.8548 & 7.8548 & 7.8548 & 7.8548 \\
& II & 7.8550 & 7.8554 & 7.8560 & 7.8573 \\
& III & 7.8555 & 7.8567 & 7.8586 & 7.8625 \\
\hline
\end{tabular}

It is interesting to note that with the complete breakage in the lacing wire, one of the values among the cluster of three values gets repeated for all the submodes as shown in the last column of Tables $3,4,5$, and 6 . The system with broken lacing wire gets converted to two configurations, that is, one with stand-alone blade and the other with two blades connected with lacing wire as shown in Figure 10. Thus, the first submode frequency of the fundamental mode appears twice for the two subsystems created due to brakeage of lacing wire connection. This is revealed clearly in Figures 11(a)-11(d), where the second submode frequency converges to the first submode frequency. This finding may be used for detecting complete breakage of lacing wire.

\section{Lacing Wire Connection within the Span of Blades}

In order to investigate the effect of lacing wire location on the natural frequencies of grouped blades, the study is further extended for the three blade packets with the lacing wire connected at a location inside the span of the blade as shown in Figure 12.

The analytical model formulation is carried out in a way similar to that for lacing wires at the tip, with the exception 


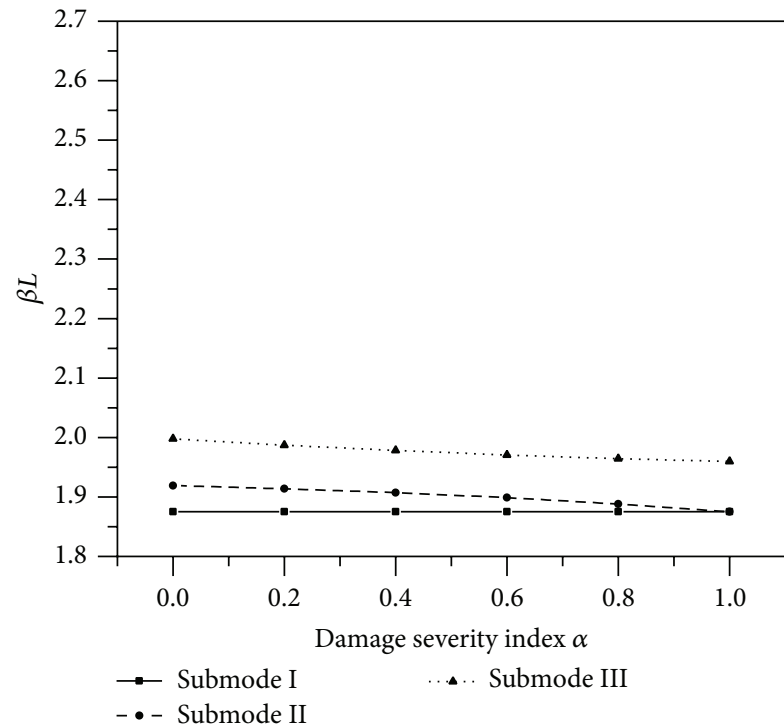

(a)

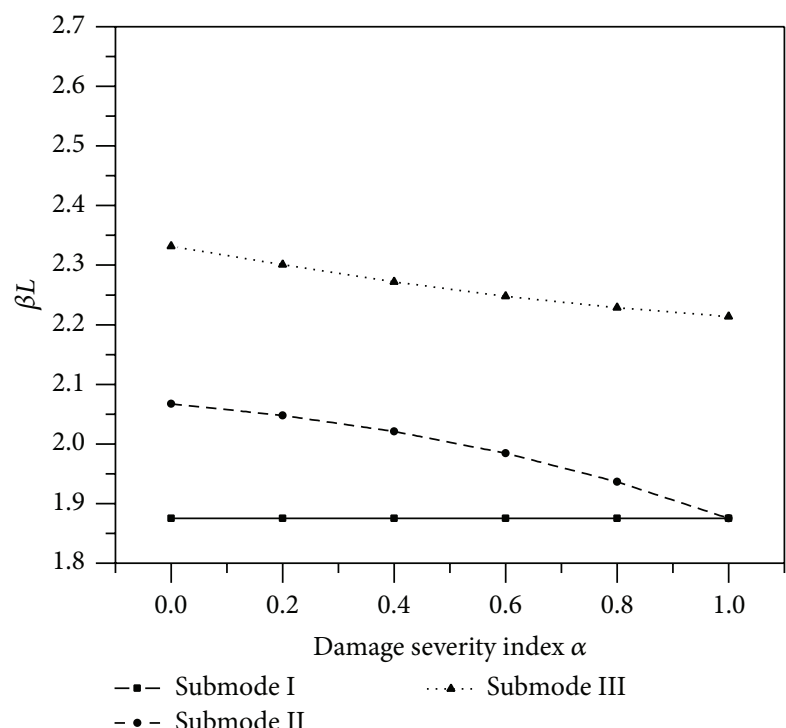

(c)

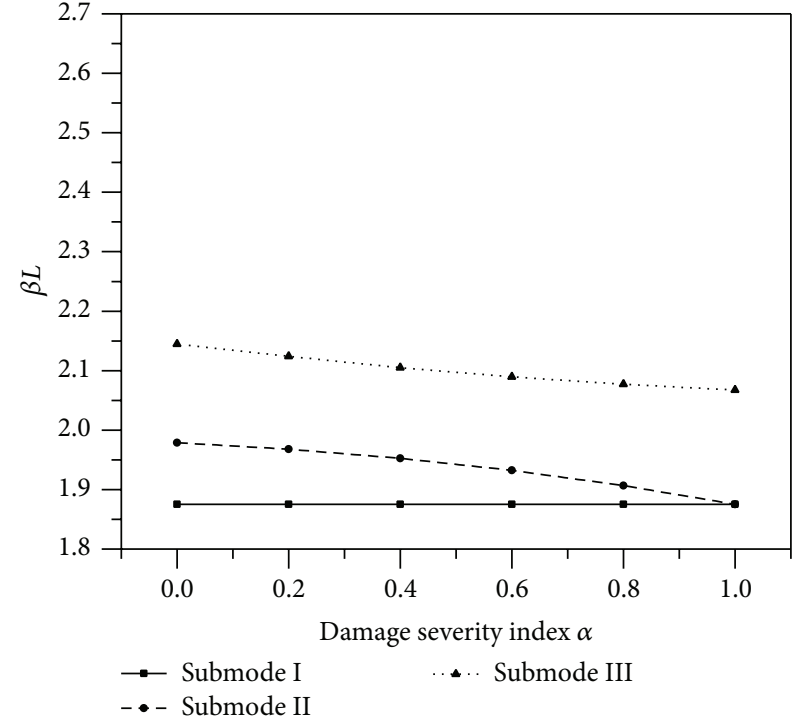

(b)

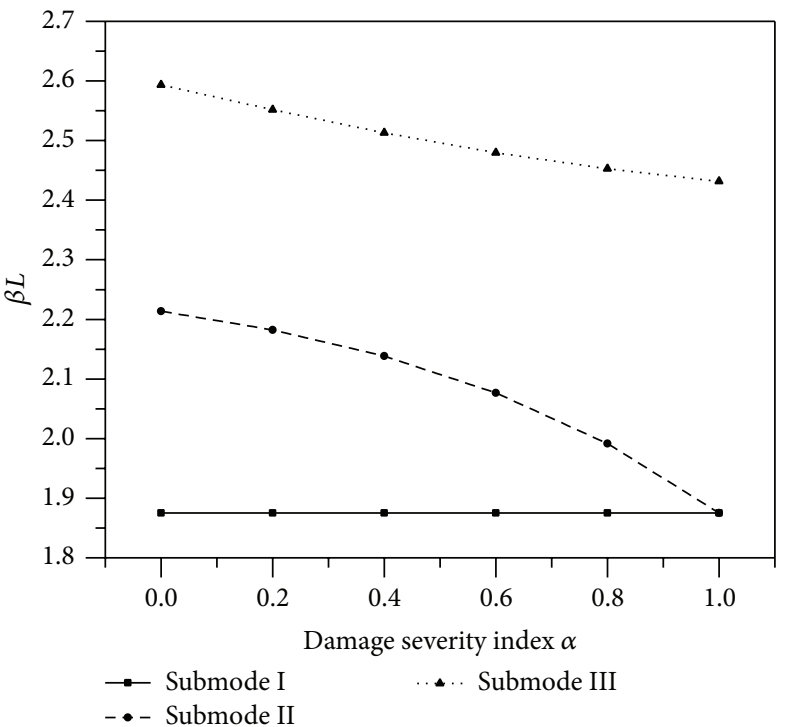

(d)

FIGURE 11: Mistuning effect on frequency of submodes of packet of blades with lacing wire at the blade tip for (a) $\lambda=0.1,(\mathrm{~b}) \lambda=0.25$, (c) $\lambda=$ 0.5 , and (d) $\lambda=1.0$.

that each beam is divided into two beams at the interface of the location of the lacing wire spring connection with the beam. Thus, each of the blades has two segments and eight constants in the mode shape description, with four in each segment. These coefficients are reduced to four in number, employing the four boundary conditions: two at blade root and another two at blade tip. There are four equations due to compatibility conditions at lacing wire location for each of the blades and as a result there are twelve equations and twelve unknowns to be obtained for the grouped system. For nontrivial solution, the determinant of the coefficients is zero and the expansion of the determinant leads to the characteristic equation.

BC's are as follows for the upper blade's left segment of length " $a$ " at $x_{1}=0, y_{1}\left(x_{1}\right)=0$ and $y_{1}^{\prime}\left(x_{1}\right)=0$; therefore, $C_{3}=$ $-C_{1}$ and $C_{4}=-C_{2}$.

For the right segment of length " $b$ "

at $x_{2}=0, y_{2}^{\prime \prime}\left(x_{2}\right)=0$ and $y_{2}^{\prime \prime \prime}\left(x_{2}\right)=0$; therefore, $C_{7}=C_{5}$ and $C_{8}=C_{6}$.

Similarly, for middle and lower blades, we get

$$
\begin{aligned}
& C_{11}=-C_{9}, \quad C_{12}=-C_{10}, \quad C_{15}=C_{13}, \\
& C_{16}=C_{14}, \\
& C_{19}=-C_{17}, \quad C_{20}=-C_{18}, \quad C_{23}=C_{21}, \\
& C_{24}=C_{22} .
\end{aligned}
$$


However four equations due to compatibility conditions of displacement, slope, BM, and SF at the location of lacing wire connection for the upper blade are as follows:

$$
\begin{gathered}
Y_{1(a)}=Y_{2(-b)}, \quad Y_{1(a)}^{\prime}=Y_{2(-b)}^{\prime}, \\
Y_{1(a)}^{\prime \prime}=Y_{2(-b)}^{\prime \prime}, \\
Y_{1(a)}^{\prime \prime \prime}=Y_{2(-b)}^{\prime \prime \prime}+k_{1} Y_{1(a)}, \\
C_{1}(\cosh \beta a-\cos \beta a)+C_{2}(\sinh \beta a-\sin \beta a) \\
-C_{5}(\cosh \beta b-\cos \beta b)-C_{6}(-\sinh \beta b-\sin \beta b)=0, \\
C_{1}(\sinh \beta a+\sin \beta a)+C_{2}(\cosh \beta a-\cos \beta a) \\
-C_{5}(-\sinh \beta b+\sin \beta b)-C_{6}(\cosh \beta b+\cos \beta b)=0 \\
C_{1}(\cosh \beta a+\cos \beta a)+C_{2}(\sinh \beta a+\sin \beta a) \\
-C_{5}(\cosh \beta b-\cos \beta b)-C_{6}(-\sinh \beta b+\sin \beta b)=0 \\
C_{1}\left[(\sinh \beta a-\sin \beta a)-(\cosh \beta a-\cos \beta a) \frac{k_{1}}{\operatorname{EI} \beta^{3}}\right] \\
+C_{10}(\sinh \beta a-\sin \beta a) \frac{k_{1}}{\operatorname{EI} \beta^{3}}=0 . \\
+C_{2}\left[(\cosh \beta a+\cos \beta a)-(\sinh \beta a-\sin \beta a) \frac{k_{1}}{\operatorname{EI} \beta^{3}}\right] \\
-C_{5}(-\sinh \beta b-\sin \beta b)-C_{6}(\cosh \beta b-\cos \beta b) \\
\operatorname{EI} \beta_{9}^{3}(\cosh \beta a-\cos \beta a) \\
k_{1}
\end{gathered}
$$

\begin{tabular}{|c|c|c|c|c|c|c|c|c|c|c|c|}
\hline$R_{a}$ & $S_{a}$ & $-P_{b}$ & $Q_{b}$ & 0 & 0 & 0 & 0 & 0 & 0 & 0 & 0 \\
\hline$Q_{a}$ & $R_{a}$ & $S_{b}$ & $-P_{b}$ & 0 & 0 & 0 & 0 & 0 & 0 & 0 & 0 \\
\hline$P_{a}$ & $Q_{a}$ & $-R_{b}$ & $S_{b}$ & 0 & 0 & 0 & 0 & 0 & 0 & 0 & 0 \\
\hline$S_{a}-R_{a} \frac{\lambda_{1}}{(\beta L)^{3}}$ & $P_{a}-S_{a} \frac{\lambda_{1}}{(\beta L)^{3}}$ & $Q_{b}$ & $-R_{b}$ & $R_{a} \frac{\lambda_{1}}{(\beta L)^{3}}$ & $S_{a} \frac{\lambda_{1}}{(\beta L)^{3}}$ & 0 & 0 & 0 & 0 & 0 & 0 \\
\hline 0 & 0 & 0 & 0 & $R_{a}$ & $S_{a}$ & $-P_{b}$ & $Q_{b}$ & 0 & 0 & 0 & 0 \\
\hline 0 & 0 & 0 & 0 & $Q_{a}$ & $R_{a}$ & $S_{b}$ & $-P_{b}$ & 0 & 0 & 0 & 0 \\
\hline 0 & 0 & 0 & 0 & $P_{a}$ & $Q_{a}$ & $-R_{b}$ & $S_{b}$ & 0 & 0 & 0 & 0 \\
\hline$R_{a} \frac{\lambda_{1}}{(\beta L)^{3}}$ & $S_{a} \frac{\lambda_{1}}{(\beta L)^{3}}$ & 0 & 0 & $S_{a}-R_{a} \frac{\lambda_{1}+\lambda_{2}}{(\beta L)^{3}}$ & $P_{a}-S_{a} \frac{\lambda_{1}+\lambda_{2}}{(\beta L)^{3}}$ & $Q_{b}$ & $-R_{b}$ & $R_{a} \frac{\lambda_{2}}{(\beta L)^{3}}$ & $S_{a} \frac{\lambda_{2}}{(\beta L)^{3}}$ & 0 & 0 \\
\hline 0 & 0 & 0 & 0 & 0 & 0 & 0 & 0 & $R_{a}$ & $S_{a}$ & $-P_{b}$ & $Q_{b}$ \\
\hline 0 & 0 & 0 & 0 & 0 & 0 & 0 & 0 & $Q_{a}$ & $R_{a}$ & $S_{b}$ & $-P_{b}$ \\
\hline 0 & 0 & 0 & 0 & 0 & 0 & 0 & 0 & $P_{a}$ & $Q_{a}$ & $-R_{b}$ & $S_{b}$ \\
\hline 0 & 0 & 0 & 0 & $R_{a} \frac{\lambda_{2}}{(\beta L)^{3}}$ & $S_{a} \frac{\lambda_{2}}{(\beta L)^{3}}$ & 0 & 0 & $S_{a}-R_{a} \frac{\lambda_{2}}{(\beta L)^{3}}$ & $P_{a}-S_{a} \frac{\lambda_{2}}{(\beta L)^{3}}$ & $Q_{b}$ & $-R_{b}$ \\
\hline
\end{tabular}

The first three equations of compatibility conditions are the same for the middle and lower blades, whereas (16) of shear force are different and are given as follows:

$$
\begin{aligned}
& C_{1}(\cosh \beta a-\cos \beta a) \frac{k_{1}}{\mathrm{EI} \beta^{3}}+C_{2}(\sinh \beta a-\sin \beta a) \frac{k_{1}}{\mathrm{EI} \beta^{3}} \\
& +C_{9}\left[(\sinh \beta a-\sin \beta a)-(\cosh \beta a-\cos \beta a) \frac{k_{1}+k_{2}}{\mathrm{EI} \beta^{3}}\right] \\
& +C_{10}\left[(\cosh \beta a+\cos \beta a)-(\sinh \beta a-\sin \beta a) \frac{k_{1}+k_{2}}{\mathrm{EI} \beta^{3}}\right] \\
& -C_{13}(-\sinh \beta b-\sin \beta b)-C_{14}(\cosh \beta b-\cos \beta b) \\
& +C_{17}(\cosh \beta a-\cos \beta a) \frac{k_{2}}{\mathrm{EI} \beta^{3}} \\
& +C_{18}(\sinh \beta a-\sin \beta a) \frac{k_{2}}{\mathrm{EI} \beta^{3}}=0, \\
& C_{9}(\cosh \beta a-\cos \beta a) \frac{k_{2}}{\mathrm{EI} \beta^{3}}+C_{10}(\sinh \beta a-\sin \beta a) \frac{k_{2}}{\mathrm{EI} \beta^{3}} \\
& +C_{17}\left[(\sinh \beta a-\sin \beta a)-(\cosh \beta a-\cos \beta a) \frac{k_{2}}{\mathrm{EI} \beta^{3}}\right] \\
& +C_{18}\left[(\cosh \beta a+\cos \beta a)-(\sinh \beta a-\sin \beta a) \frac{k_{2}}{\mathrm{EI} \beta^{3}}\right] \\
& +C_{21}(-\sinh \beta b-\sin \beta b)-C_{22}(\cosh \beta b-\cos \beta b)=0 .
\end{aligned}
$$

These equations can be arranged to get frequency equation in determinant form as 


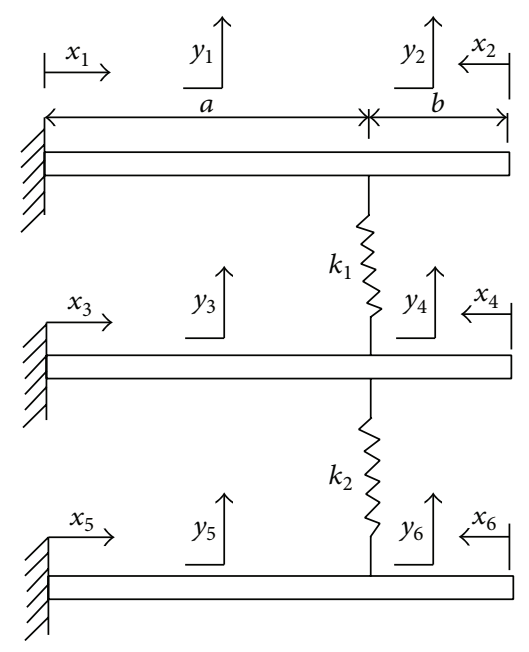

FIGURE 12: Three blades interconnected with lacing wire inside the span.

TABLE 8: Effect of lacing wire damage induced mistuning (for $\lambda=0.1$ ) on natural frequencies of the blade packet (lacing wire at 2/3rd of blade span).

\begin{tabular}{|c|c|c|c|c|c|c|c|}
\hline \multirow{2}{*}{ Mode } & \multirow{2}{*}{ Submode } & \multicolumn{6}{|c|}{$\beta L$ values } \\
\hline & & $\alpha=0$ & $\alpha=0.2$ & $\alpha=0.4$ & $\alpha=0.6$ & $\alpha=0.8$ & $\alpha=1.0$ \\
\hline \multirow{3}{*}{1} & I & 1.8751 & 1.8751 & 1.8751 & 1.8751 & 1.8751 & 1.8751 \\
\hline & II & 1.8886 & 1.8870 & 1.8849 & 1.8823 & 1.8790 & 1.8751 \\
\hline & III & 1.9146 & 1.9110 & 1.9078 & 1.9053 & 1.9032 & 1.9017 \\
\hline \multirow{3}{*}{2} & I & 4.6941 & 4.6941 & 4.6941 & 4.6941 & 4.6941 & 4.6941 \\
\hline & II & 4.6946 & 4.6946 & 4.6945 & 4.6944 & 4.6942 & 4.6941 \\
\hline & III & 4.6957 & 4.6955 & 4.6954 & 4.6953 & 4.6952 & 4.6951 \\
\hline \multirow{3}{*}{3} & I & 7.8548 & 7.8548 & 7.8548 & 7.8548 & 7.8548 & 7.8548 \\
\hline & II & 7.8550 & 7.8550 & 7.8549 & 7.8549 & 7.8548 & 7.8548 \\
\hline & III & 7.8555 & 7.8555 & 7.8554 & 7.8553 & 7.8553 & 7.8553 \\
\hline
\end{tabular}

TABLE 9: Effect of lacing wire damage induced mistuning (for $\lambda=0.25$ ) on natural frequencies (lacing wire at 2/3rd of blade span).

\begin{tabular}{cccccccc}
\hline \multirow{2}{*}{ Mode } & \multirow{2}{*}{ Submode } & $\alpha=0$ & $\alpha=0.2$ & $\alpha=0.4$ & $\alpha=0.6$ & $\alpha=0.8$ & $\alpha=1.0$ \\
\hline \multirow{3}{*}{1} & I & 1.8751 & 1.8751 & 1.8751 & 1.8751 & 1.8751 & 1.8751 \\
& II & 1.9082 & 1.9044 & 1.8994 & 1.8928 & 1.8847 & 1.8751 \\
& III & 1.9694 & 1.9611 & 1.9538 & 1.9478 & 4.9431 & 4.6941 \\
2 & I & 4.6941 & 4.6941 & 4.6941 & 4.6941 & 4.6941 \\
& II & 4.6954 & 4.6952 & 4.6950 & 4.6948 & 4.6941 \\
3 & III & 4.6980 & 4.6976 & 4.6973 & 4.6970 & 4.6968 & 4.6967 \\
\hline
\end{tabular}

where $P_{a}=\cosh \beta L \mu+\cos \beta L \mu$, in which $\mu=a / L$ is normalized lacing wire location from the root, $Q_{a}=\sinh \beta L \mu+$ $\sin \beta L \mu, R_{a}=\cosh \beta L \mu-\cos \beta L \mu$, and $S_{a}=\sinh \beta L \mu-$ $\sin \beta L \mu$ and $P_{b}=\cosh \beta L(1-\mu)+\cos \beta L(1-\mu)$, in which $1-\mu=b / L, Q_{b}=\sinh \beta L(1-\mu)+\sin \beta L(1-\mu), R_{b}=$ $\cosh \beta L(1-\mu)-\cos \beta L(1-\mu)$, and $S_{b}=\sinh \beta L(1-\mu)-$ $\sin \beta L(1-\mu)$.

5.1. Lacing Wire Connection at 2/3rd of the Blade Span. The first intermediate position for connection of lacing wire 


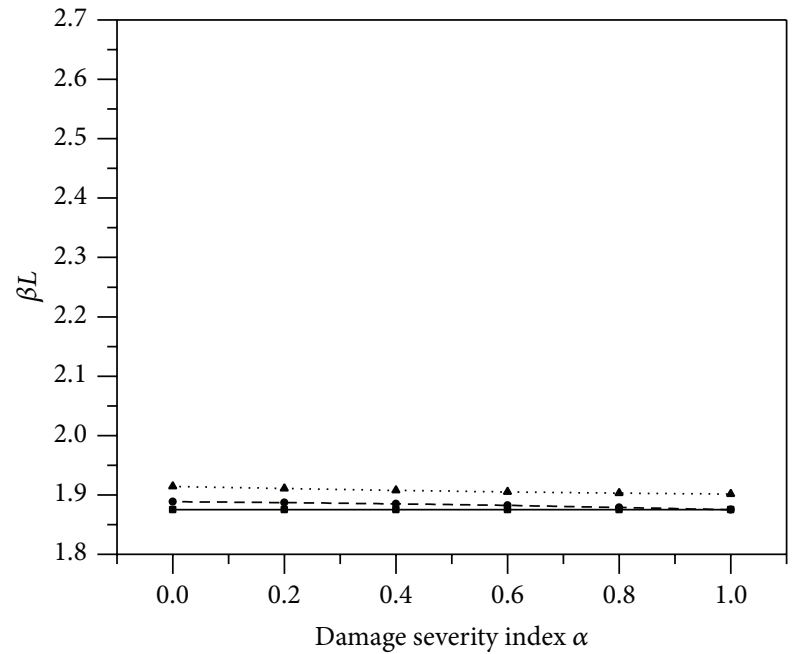

$\rightarrow-$ Submode I

- - Submode II

(a)

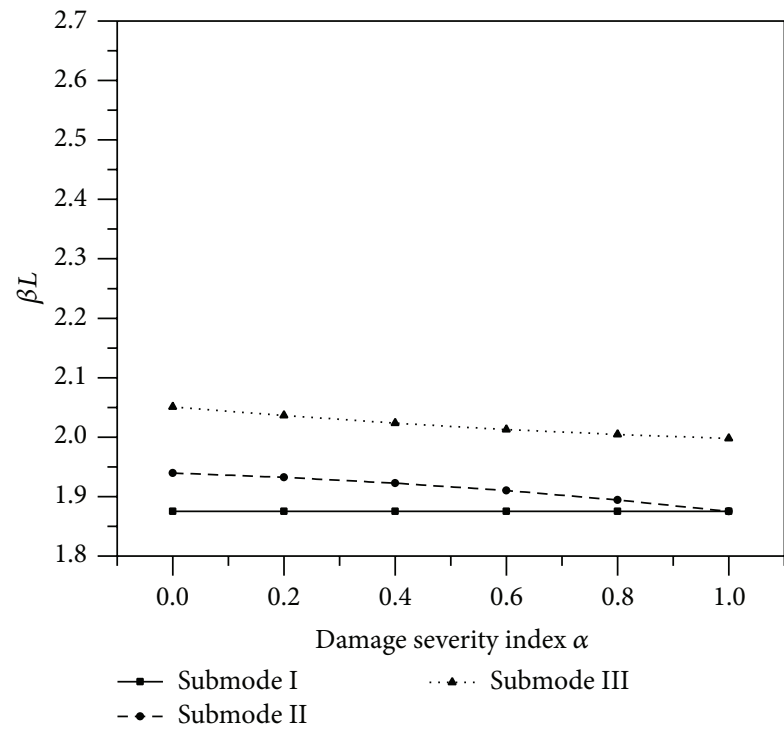

(c)

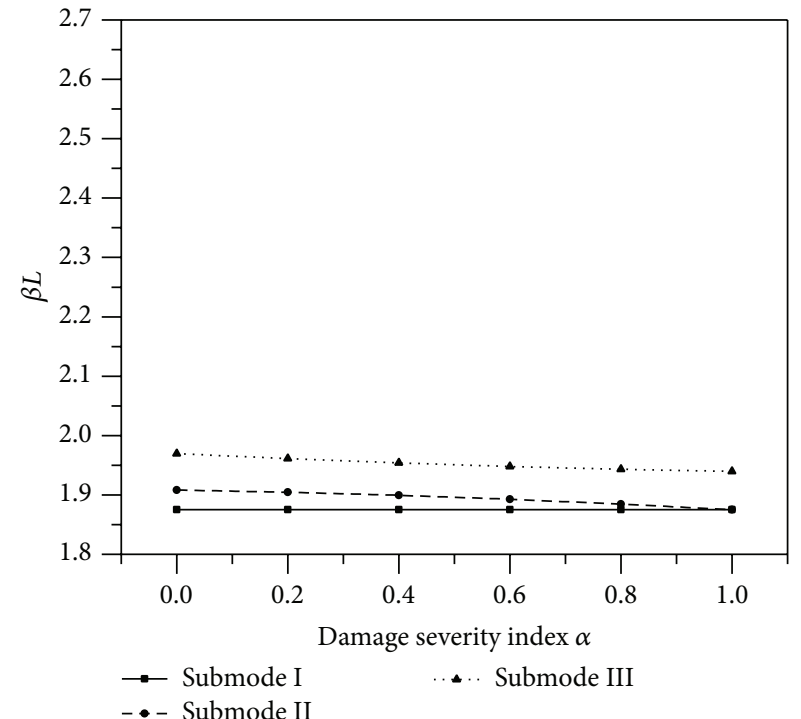

(b)

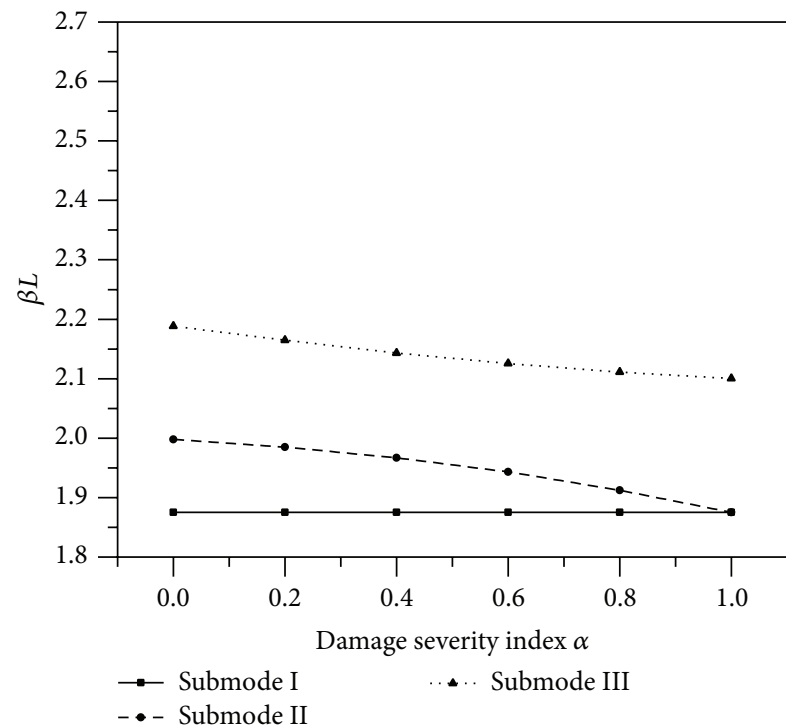

(d)

FIGURE 13: Mistuning effect on frequency of submodes of packet of blades with lacing wire at $2 / 3$ rd of the blade span for (a) $\lambda=0.1$, (b) $\lambda=$ 0.25 , (c) $\lambda=0.5$, and (d) $\lambda=1.0$.

TABLE 10: Effect of lacing wire damage induced mistuning (for $\lambda=0.5$ ) on natural frequencies (lacing wire at 2/3rd of blade span).

\begin{tabular}{|c|c|c|c|c|c|c|c|}
\hline \multirow{2}{*}{ Mode } & \multirow{2}{*}{ Submode } & \multicolumn{6}{|c|}{$\beta L$ values } \\
\hline & & $\alpha=0$ & $\alpha=0.2$ & $\alpha=0.4$ & $\alpha=0.6$ & $\alpha=0.8$ & $\alpha=1.0$ \\
\hline \multirow{3}{*}{1} & I & 1.8751 & 1.8751 & 1.8751 & 1.8751 & 1.8751 & 1.8751 \\
\hline & II & 1.9396 & 1.9324 & 1.9227 & 1.9100 & 1.8941 & 1.8751 \\
\hline & III & 2.0511 & 2.0364 & 2.0235 & 2.0128 & 2.0043 & 1.9978 \\
\hline \multirow{3}{*}{2} & I & 4.6941 & 4.6941 & 4.6941 & 4.6941 & 4.6941 & 4.6941 \\
\hline & II & 4.6967 & 4.6964 & 4.6960 & 4.6955 & 4.6948 & 4.6941 \\
\hline & III & 4.7019 & 4.7012 & 4.7005 & 4.7000 & 4.6996 & 4.6993 \\
\hline \multirow{3}{*}{3} & I & 7.8548 & 7.8548 & 7.8548 & 7.8548 & 7.8548 & 7.8548 \\
\hline & II & 7.8560 & 7.8559 & 7.8557 & 7.8554 & 7.8551 & 7.8548 \\
\hline & III & 7.8586 & 7.8583 & 7.8579 & 7.8577 & 7.8575 & 7.8573 \\
\hline
\end{tabular}


TABLE 11: Effect of lacing wire damage induced mistuning (for $\lambda=1$ ) on natural frequencies (lacing wire at 2/3rd of blade span).

\begin{tabular}{|c|c|c|c|c|c|c|c|}
\hline \multirow{2}{*}{ Mode } & \multirow{2}{*}{ Submode } & \multicolumn{6}{|c|}{$\beta L$ values } \\
\hline & & $\alpha=0$ & $\alpha=0.2$ & $\alpha=0.4$ & $\alpha=0.6$ & $\alpha=0.8$ & $\alpha=1.0$ \\
\hline \multirow{3}{*}{1} & I & 1.8751 & 1.8751 & 1.8751 & 1.8751 & 1.8751 & 1.8751 \\
\hline & II & 1.9978 & 1.9847 & 1.9668 & 1.9430 & 1.9125 & 1.8751 \\
\hline & III & 2.1884 & 2.1646 & 2.1433 & 2.1254 & 2.1111 & 2.1002 \\
\hline \multirow{3}{*}{2} & I & 4.6941 & 4.6941 & 4.6941 & 4.6941 & 4.6941 & 4.6941 \\
\hline & II & 4.6993 & 4.6987 & 4.6979 & 4.6968 & 4.6956 & 4.6941 \\
\hline & III & 4.7098 & 4.7083 & 4.7070 & 4.7060 & 4.7052 & 4.7045 \\
\hline \multirow{3}{*}{3} & I & 7.8548 & 7.8548 & 7.8548 & 7.8548 & 7.8548 & 7.8548 \\
\hline & II & 7.8573 & 7.8570 & 7.8566 & 7.8561 & 7.8555 & 7.8548 \\
\hline & III & 7.8625 & 7.8617 & 7.8611 & 7.8606 & 7.8602 & 7.8599 \\
\hline
\end{tabular}

TABLE 12: Natural frequencies ( $\beta L$ values) of the tuned blade packet with varying stiffness ratios (lacing wire at mid span).

\begin{tabular}{lllllr}
\hline Mode & Submode & $\lambda=0.1$ & $\lambda=0.25$ & $\lambda=0.5$ & 1.8751 \\
1 & I & 1.8751 & 1.8751 & 1.9006 & 1.8751 \\
& II & 1.8803 & 1.8880 & 1.9479 & 2.9249 \\
\hline \multirow{3}{*}{2} & III & 1.8906 & 1.9129 & 4.6941 & 4.0109 \\
\hline & I & 4.6941 & 4.6941 & 4.7015 & 4.6941 \\
3 & II & 4.6956 & 4.6978 & 4.7162 & 7.7275 \\
\hline & III & 4.6985 & 4.7052 & 7.8548 & 7.8548 \\
& I & 7.8548 & 7.8548 & 7.8548 & 7.8548 \\
\hline
\end{tabular}

TABLE 13: Effect of lacing wire damage induced mistuning (for $\lambda=0.1$ ) on natural frequencies (lacing wire at mid span).

\begin{tabular}{|c|c|c|c|c|c|c|c|}
\hline \multirow{2}{*}{ Mode } & \multirow{2}{*}{ Submode } & \multicolumn{6}{|c|}{$\beta L$ values } \\
\hline & & $\alpha=0$ & $\alpha=0.2$ & $\alpha=0.4$ & $\alpha=0.6$ & $\alpha=0.8$ & $\alpha=1.0$ \\
\hline \multirow{3}{*}{1} & I & 1.8751 & 1.8751 & 1.8751 & 1.8751 & 1.8751 & 1.8751 \\
\hline & II & 1.8803 & 1.8797 & 1.8789 & 1.8779 & 1.8766 & 1.8751 \\
\hline & III & 1.8906 & 1.8891 & 1.8879 & 1.8869 & 1.8861 & 1.8855 \\
\hline \multirow{3}{*}{2} & I & 4.6941 & 4.6941 & 4.6941 & 4.6941 & 4.6941 & 4.6941 \\
\hline & II & 4.6956 & 4.6954 & 4.6952 & 4.6949 & 4.6945 & 4.6941 \\
\hline & III & 4.6985 & 4.6981 & 4.6977 & 4.6975 & 4.6972 & 4.6970 \\
\hline \multirow{3}{*}{3} & I & 7.8548 & 7.8548 & 7.8548 & 7.8548 & 7.8548 & 7.8548 \\
\hline & II & 7.8548 & 7.8548 & 7.8548 & 7.8548 & 7.8548 & 7.8548 \\
\hline & III & 7.8548 & 7.8548 & 7.8548 & 7.8548 & 7.8548 & 7.8548 \\
\hline
\end{tabular}

is considered as $2 / 3$ rd of the blade span (Figure 13) and accordingly normalized length of the two segments is used in the frequency equation to get the set of values indicated in Table 7 .

It is observed from Table 7 that natural frequencies of all the submodes are reducing with the shifting of location of lacing wire towards the blade root. This observation is quite obvious as the ratio of lacing wire stiffness to blade stiffness $\lambda$ will be reducing with the shifting of lacing wires towards the root of the blade compared to the ratio when lacing wires are at the tip of blade. This is due to the increase in stiffness of the blade with the shifting of spring force towards the fixed end compared to spring force at free end. Therefore, smaller value of stiffness ratio goes into calculation for natural frequencies resulting in a drop in natural frequencies.

It is also evident that the reduction in the fundamental mode frequencies is more compared to the subsequent modes. This is due to the fact that the system stiffness increases in higher modes and as a result there is a drop in $\lambda$. Moreover, the higher frequencies of submodes in the cluster are more sensitive than earlier, as lacing wire stiffness contribution is more in higher submodes. 


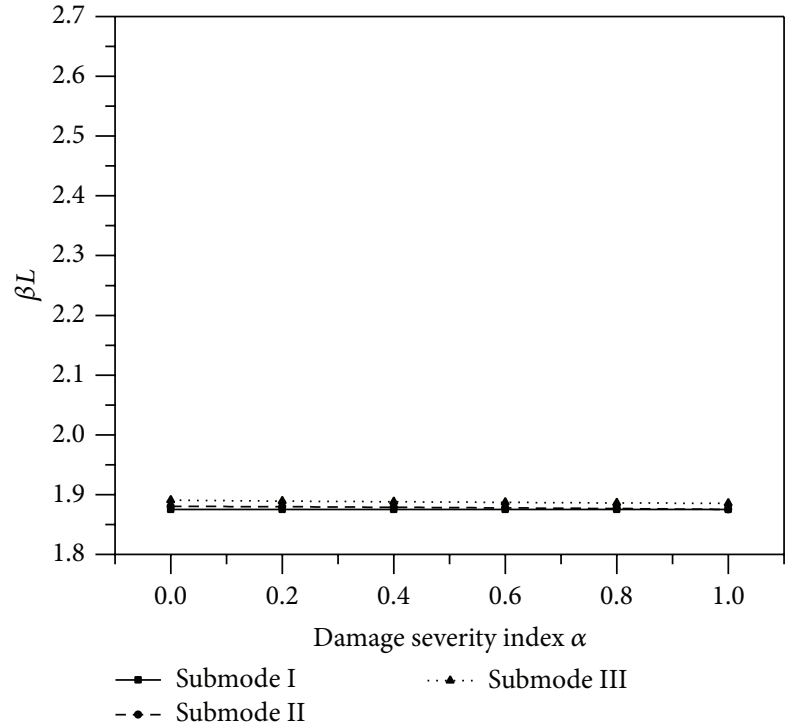

(a)

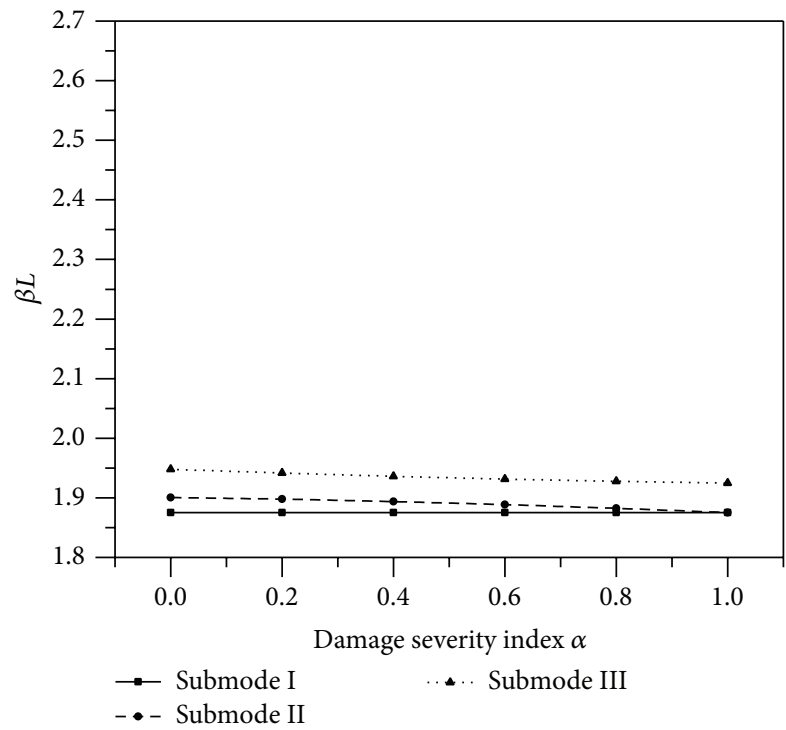

(c)

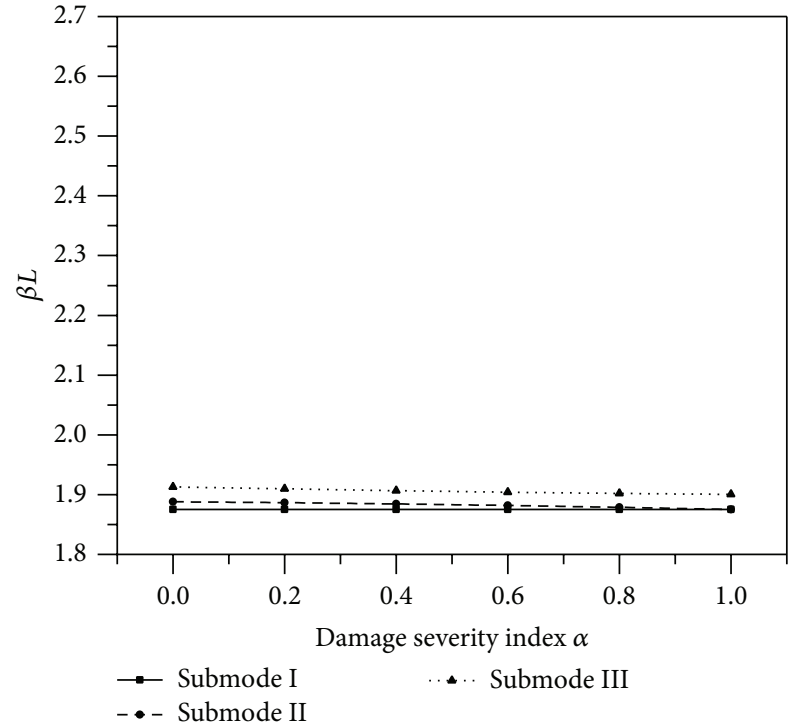

(b)

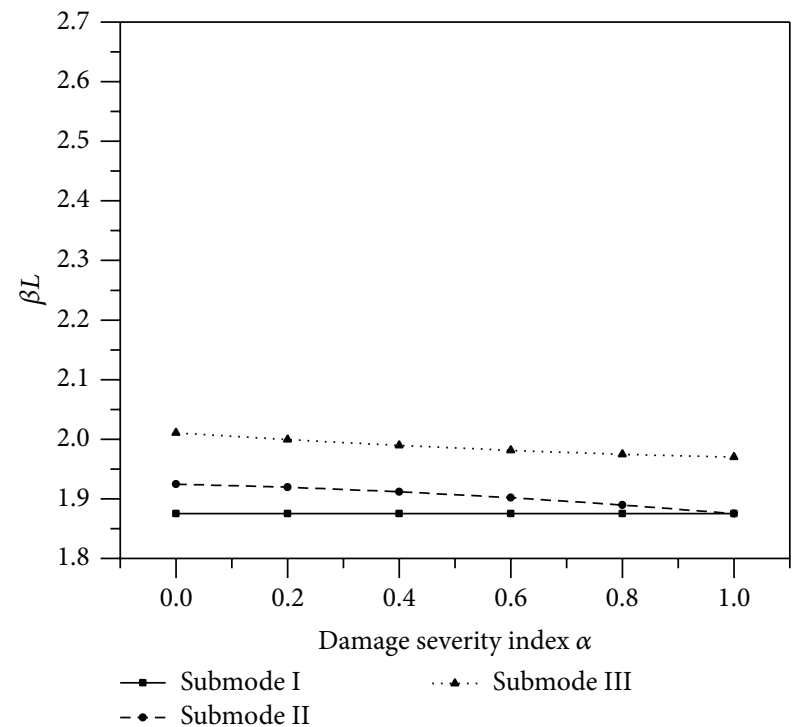

(d)

Figure 14: Mistuning effect on frequency of submodes of packet of blades with lacing wire at middle of the blade span for (a) $\lambda=0.1$, (b) $\lambda=$ 0.25 , (c) $\lambda=0.5$, and (d) $\lambda=1.0$.

It is also observed that the first submode frequency in all the modes does not change, whereas other frequencies are getting changed albeit for higher modes the change is the least. On the basis of this result, it is concluded that only submodes of the fundamental mode can be used as an indicator for lacing wire stiffness reduction.

5.2. Lacing Wire Connection at the Middle of Blade Span. As observed in the above section, natural frequency of all the submodes decreases with shifting in location of lacing wire connection towards blade root. It is the fundamental mode which is observed to be more sensitive to change in location. To investigate the effect of connection on antinode and node points of various modes, this study is extended with lacing wire connection at the middle of the blade which is close to the antinode of the second mode of vibration (Figure 14). Table 12 presents the effect of varying stiffness ratio $\lambda$ on natural frequency of submodes of three modes of vibration.

It is clear from Table 12 that the submodes of the second mode also get affected with the rise in stiffness ratio value like the submodes of the fundamental mode, whereas submodes of the third mode remain unaffected.

5.3. General Comparison. Finally, a comparative picture of effect of lacing wire damage on the change in natural frequencies for all three lacing wire positions considered here 


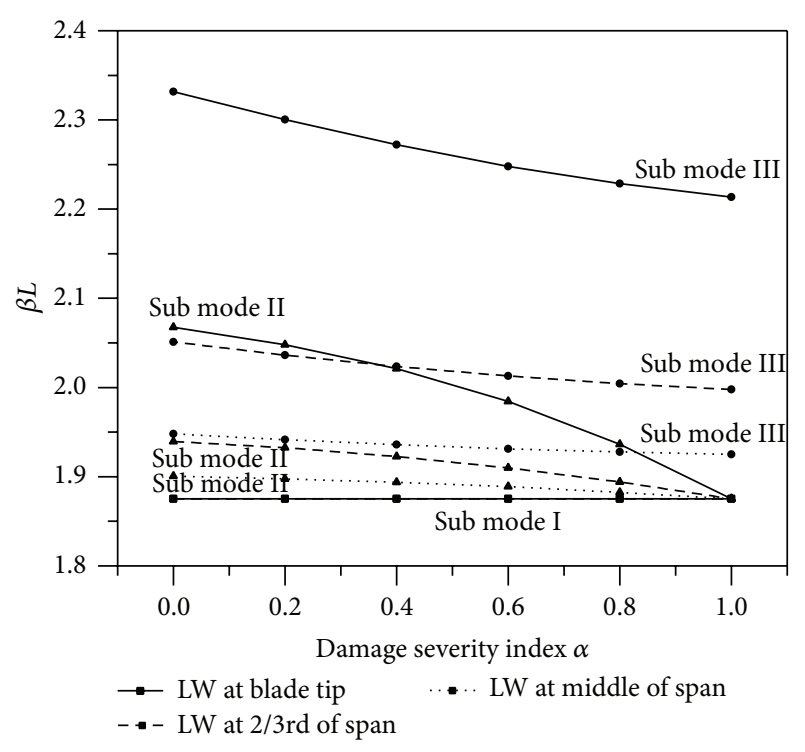

FIGURE 15: Comparison of lacing wire damage induced mistuning in the three different tuned systems with the stiffness ratio of lacing wire $\lambda=0.5$.

TABLE 14: Effect of lacing wire damage induced mistuning (for $\lambda=$ 0.25 ) on natural frequencies (lacing wire at mid span).

\begin{tabular}{llllllll}
\hline \multirow{2}{*}{ Mode Submode } & \multicolumn{6}{c}{$\beta L$ values } \\
& & $\alpha=0$ & $\alpha=0.2$ & $\alpha=0.4$ & $\alpha=0.6$ & $\alpha=0.8$ & $\alpha=1.0$ \\
\hline \multirow{3}{*}{1} & I & 1.8751 & 1.8751 & 1.8751 & 1.8751 & 1.8751 & 1.8751 \\
& II & 1.8880 & 1.8866 & 1.8846 & 1.8820 & 1.8788 & 1.8751 \\
& III & 1.9129 & 1.9095 & 1.9065 & 1.9040 & 1.9021 & 1.9006 \\
\hline \multirow{3}{*}{2} & I & 4.6941 & 4.6941 & 4.6941 & 4.6941 & 4.6941 & 4.6941 \\
& II & 4.6978 & 4.6974 & 4.6968 & 4.6960 & 4.6951 & 4.6941 \\
& III & 4.7052 & 4.7041 & 4.7032 & 4.7025 & 4.7019 & 4.7015 \\
\hline \multirow{3}{*}{3} & I & 7.8548 & 7.8548 & 7.8548 & 7.8548 & 7.8548 & 7.8548 \\
& II & 7.8548 & 7.8548 & 7.8548 & 7.8548 & 7.8548 & 7.8548 \\
& III & 7.8548 & 7.8548 & 7.8548 & 7.8548 & 7.8548 & 7.8548 \\
\hline
\end{tabular}

is presented in Figure 15, where it can be seen that qualitative behavior remains the same in all three cases, and irrespective of lacing wire position, natural frequencies decrease with the damage growth and the decrease is the maximum when the lacing wire is at the blade tip (Tables $8,9,10,11,13,14,15$, and 16).

\section{Conclusion}

It is established from the investigation of simplified turbine blade group dynamics and modal characteristics that splitting of natural frequencies in each mode occurs and the number of frequencies into which split occurs is equal to the number of blades forming the packet with lacing wire connections. It is found that natural frequencies of the submodes connected with fundamental mode can be used as an excellent indicator of a lacing wire damage in both qualitative and quantitative ways.
TABLE 15: Effect of lacing wire damage induced mistuning (for $\lambda=$ 0.5 ) on natural frequencies (lacing wire at mid span).

\begin{tabular}{llllllll}
\hline \multirow{2}{*}{ Mode Submode } & \multicolumn{7}{c}{$\beta L$ values } \\
& & $\alpha=0$ & $\alpha=0.2$ & $\alpha=0.4$ & $\alpha=0.6$ & $\alpha=0.8$ & $\alpha=1.0$ \\
\hline \multirow{3}{*}{1} & I & 1.8751 & 1.8751 & 1.8751 & 1.8751 & 1.8751 & 1.8751 \\
& II & 1.9006 & 1.8977 & 1.8938 & 1.8888 & 1.8825 & 1.8751 \\
& III & 1.9479 & 1.9415 & 1.9359 & 1.9312 & 1.9276 & 1.9249 \\
\hline \multirow{3}{*}{2} & I & 4.6941 & 4.6941 & 4.6941 & 4.6941 & 4.6941 & 4.6941 \\
& II & 4.7015 & 4.7006 & 4.6995 & 4.6980 & 4.6962 & 4.6941 \\
& III & 4.7162 & 4.7141 & 4.7123 & 4.7108 & 4.7097 & 4.7088 \\
\hline \multirow{3}{*}{3} & I & 7.8548 & 7.8548 & 7.8548 & 7.8548 & 7.8548 & 7.8548 \\
& II & 7.8548 & 7.8548 & 7.8548 & 7.8548 & 7.8548 & 7.8548 \\
& III & 7.8548 & 7.8548 & 7.8548 & 7.8548 & 7.8548 & 7.8548 \\
\hline
\end{tabular}

TABLE 16: Effect of lacing wire damage induced mistuning ( for $\lambda=1$ ) on natural frequencies (lacing wire at mid span).

\begin{tabular}{lllllllll}
\hline \multirow{2}{*}{ Mode Submode } & \multicolumn{7}{c}{$\beta L$ values } \\
& & $\alpha=0$ & $\alpha=0.2$ & $\alpha=0.4$ & $\alpha=0.6$ & $\alpha=0.8$ & $\alpha=1.0$ \\
\hline \multirow{3}{*}{1} & I & 1.8751 & 1.8751 & 1.8751 & 1.8751 & 1.8751 & 1.8751 \\
& II & 1.9249 & 1.9193 & 1.9118 & 1.9020 & 1.8898 & 1.8751 \\
& III & 2.0109 & 1.9996 & 1.9896 & 1.9813 & 1.9748 & 1.9698 \\
\hline \multirow{3}{*}{2} & I & 4.6941 & 4.6941 & 4.6941 & 4.6941 & 4.6941 & 4.6941 \\
& II & 4.7019 & 4.7071 & 4.7048 & 4.7019 & 4.6983 & 4.6941 \\
& III & 4.7275 & 4.7340 & 4.7304 & 4.7275 & 4.7252 & 4.7235 \\
\hline \multirow{3}{*}{3} & I & 7.8548 & 7.8548 & 7.8548 & 7.8548 & 7.8548 & 7.8548 \\
& II & 7.8548 & 7.8548 & 7.8548 & 7.8548 & 7.8548 & 7.8548 \\
& III & 7.8548 & 7.8548 & 7.8548 & 7.8548 & 7.8548 & 7.8548 \\
\hline
\end{tabular}

Another interesting observation is that though multiple frequencies remain the same in number, these frequencies spread out with stiffening of lacing wire. The effect is most prominent when the lacing wire is at the blade tip. Effect in higher mode frequencies is relatively much smaller and also depends on the position of the lacing wire with respect to the antinodes of the mode shapes. The first mode frequencies are therefore suggested to be considered for the damage diagnosis in turbine blade packet.

\section{Conflict of Interests}

The authors declare that there is no conflict of interests regarding the publication of this paper.

\section{Acknowledgments}

The author is thankful to the research review committee of the institute for its critical queries in the pursuit of answers for those queries, resulting in this fundamental work. The author also acknowledges the help extended by faculty colleague Dr. Arun kumar Singh for his encouragement in learning Mathematica and Origin software. 


\section{References}

[1] N. K. Mukhopadhyay, S. G. Chowdhury, G. Das, I. Chattoraj, S. K. Das, and D. K. Bhattacharya, "An investigation of the failure of low pressure steam turbine blades," Engineering Failure Analysis, vol. 5, no. 3, pp. 181-193, 1998.

[2] G. Gounaris and A. Dimarogonas, "A finite element of a cracked prismatic beam for structural analysis," Computers and Structures, vol. 28, no. 3, pp. 309-313, 1988.

[3] W. M. Ostachowicz and M. Krawczuk, "Analysis of the effect of cracks on the natural frequencies of a cantilever beam," Journal of Sound and Vibration, vol. 150, no. 2, pp. 191-201, 1991.

[4] P. Gudmundson, "Eigenfrequency changes of structures due to cracks, notches or other geometrical changes," Journal of the Mechanics and Physics of Solids, vol. 30, no. 5, pp. 339-353, 1982.

[5] T. G. Chondros and A. D. Dimarogonas, "Vibration of a cracked cantilever beam," Journal of Vibration and Acoustics, vol. 120, no. 3, pp. 742-746, 1998.

[6] B. P. Nandwana and S. K. Maiti, "Detection of the location and size of a crack in stepped cantilever beams based on measurements of natural frequencies," Journal of Sound and Vibration, vol. 203, no. 3, pp. 435-446, 1997.

[7] R. Tiwari and N. N. Dhawade, "Localization and criticality of a crack in an Euler-Bernoulli beam based on modal characteristics," Advances in Vibration Engineering, vol. 5, no. 3, 2006.

[8] K. H. Low, "Comparative study of the eigenvalue solutions for mass-loaded beams under classical boundary conditions," International Journal of Mechanical Sciences, vol. 43, no. 1, pp. 237-244, 2001.

[9] D. Wang, "Vibration and sensitivity analysis of a beam with a lumped mass of translational and rotary inertias," Journal of Vibration and Acoustics, Transactions of the ASME, vol. 134, no. 3, Article ID 034502, 2012.

[10] J. S. Rao, Turbomachine Blade Vibration, New Age International Publisher, 2010.

[11] D. M. Smith, "Vibration of turbine blades in packets," in Proceedings of the 7th International Congress for Applied Mechanics, p. 178, London, UK, 1948.

[12] J. P. Ellington and H. McCallion, "The vibration of laced turbine blades," Journal of the Royal Aeronautical Society, vol. 61, p. 563, 1957.

[13] M. A. Prohl, "A method for calculating vibration frequency and stress of a banded group of turbine buckets," Transactions of the ASME, vol. 80, p. 169, 1958.

[14] J. S. Rao, "Blade Group Forced Vibration-Computer Program," Tech. Memo. 76 WRL M23, Rochester Institute of Technology, Rochester, NY, USA, 1976.

[15] G. R. Bajaj, Free vibration of packeted turbine blades-coupled bending-bending-torsion modes [M.S thesis], Indian Institute of Technology Kanpur, Kanpur, India, 1974.

[16] J. Thomas and H. T. Belek, "Free vibration of blade packets," Journal of Mechanical Engineering Science, vol. 19, no. 1, p. 13, 1977.

[17] A. L. Salama and M. Petyt, "Dynamic response of packets of blades by the finite element method," Journal of Mechanical Design, vol. 100, no. 4, pp. 660-666, 1978.

[18] H. S. Lim and H. H. Yoo, "Modal analysis of a multi-blade system undergoing rotational motion," Journal of Mechanical Science and Technology, vol. 23, no. 8, pp. 2051-2058, 2009.

[19] H. S. Lim, J. Chung, and H. H. Yoo, "Modal analysis of a rotating multi-packet blade system," Journal of Sound and Vibration, vol. 325, no. 3, pp. 513-531, 2009.
[20] D. J. Ewins, "The effects of detuning upon the forced vibrations of bladed disks," Journal of Sound and Vibration, vol. 9, no. 1, pp. 65-84, 1969.

[21] S.-T. Wei and C. Pierre, "Localization phenomena in mistuned assemblies with cyclic symmetry. Part II: forced vibrations," Journal of Vibration, Acoustics, Stress, and Reliability in Design, vol. 110, no. 4, pp. 439-449, 1988.

[22] M. P. Castanier and C. Pierre, "Modeling and analysis of mistuned bladed disk vibration: status and emerging directions," Journal of Propulsion and Power, vol. 22, no. 2, pp. 384-396, 2006.

[23] J. Hou, "Cracking-induced mistuning in bladed disks," AIAA Journal, vol. 44, no. 11, pp. 2542-2546, 2006.

[24] B.-W. Huang and J.-H. Kuang, "Variation in the stability of a rotating blade disk with a local crack defect," Journal of Sound and Vibration, vol. 294, no. 3, pp. 486-502, 2006.

[25] X. Fang, J. Tang, E. Jordan, and K. D. Murphy, "Crack induced vibration localization in simplified bladed-disk structures," Journal of Sound and Vibration, vol. 291, no. 1-2, pp. 395-418, 2006.

[26] A. Saito, M. P. Castanier, and C. Pierre, "Effects of a cracked blade on mistuned turbine engine rotor vibration," Journal of Vibration and Acoustics, vol. 131, no. 6, Article ID 061006, 9 pages, 2009. 

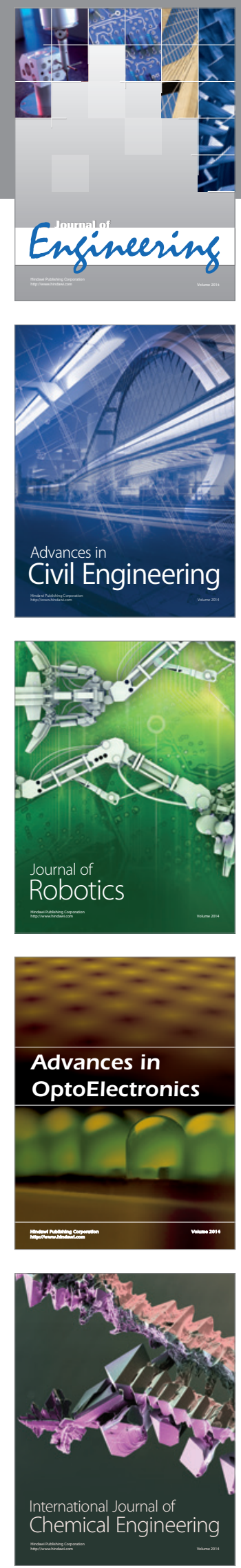

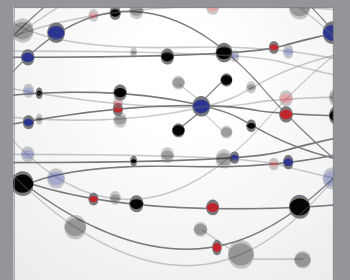

The Scientific World Journal
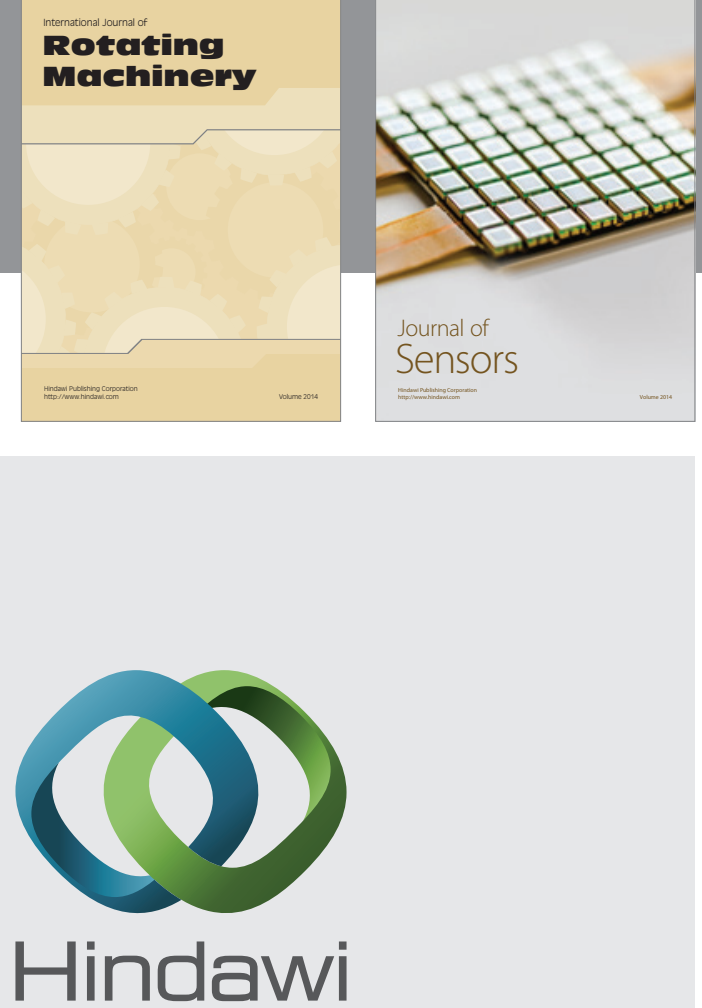

Submit your manuscripts at http://www.hindawi.com
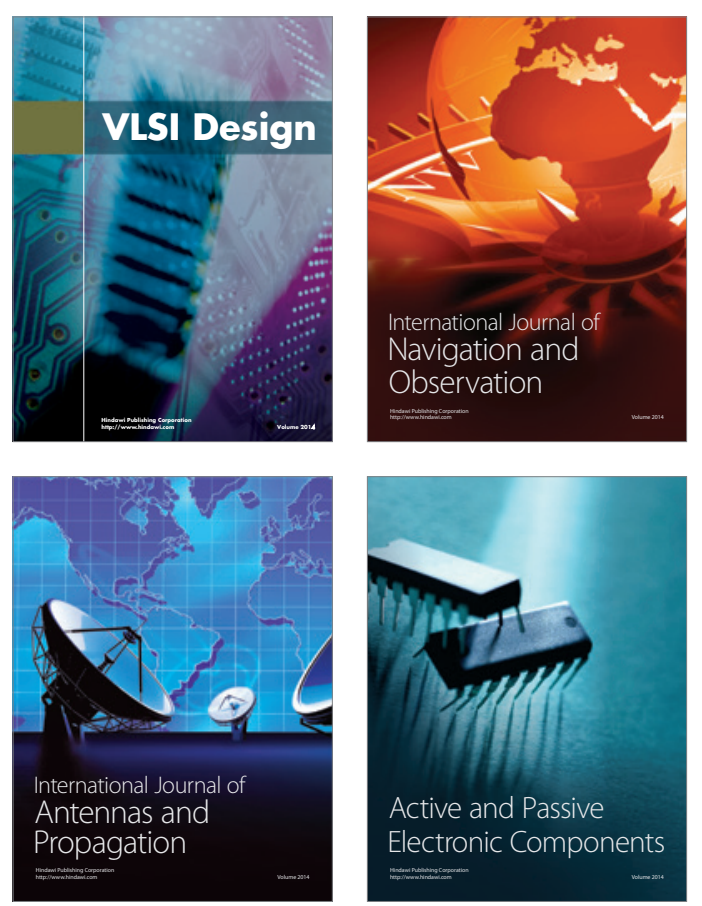
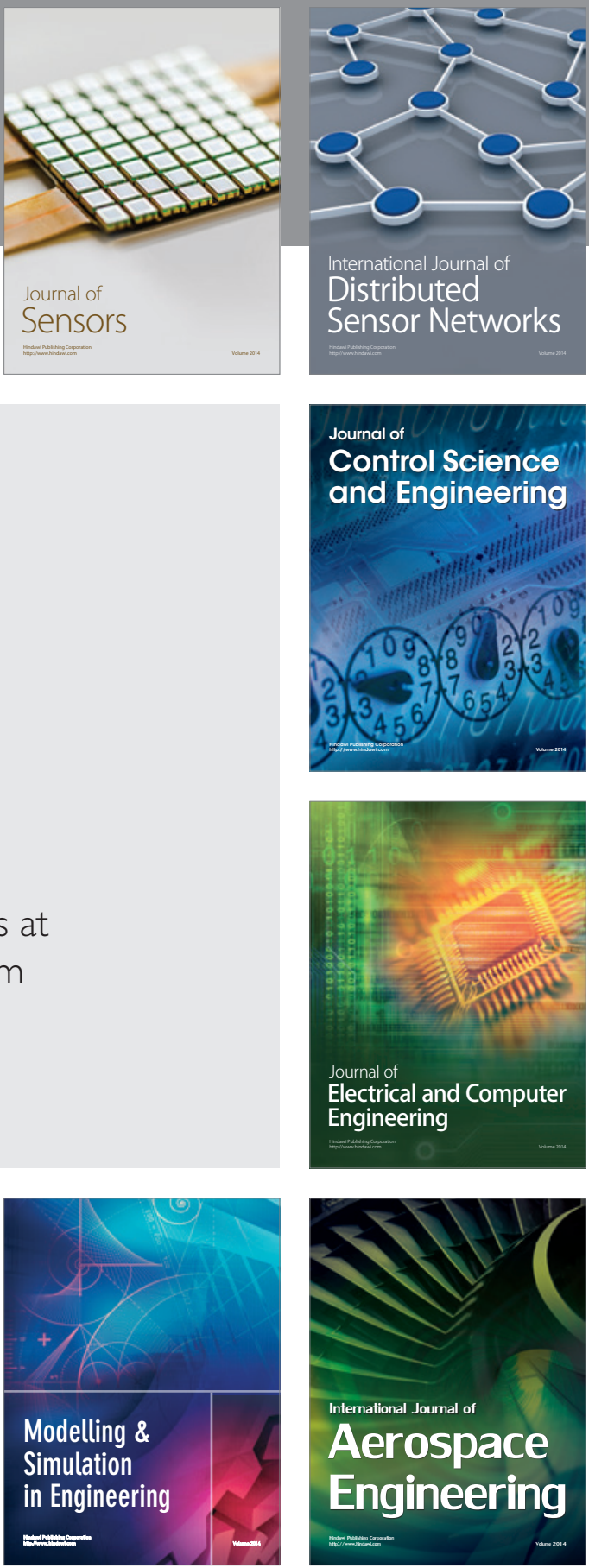

Journal of

Control Science

and Engineering
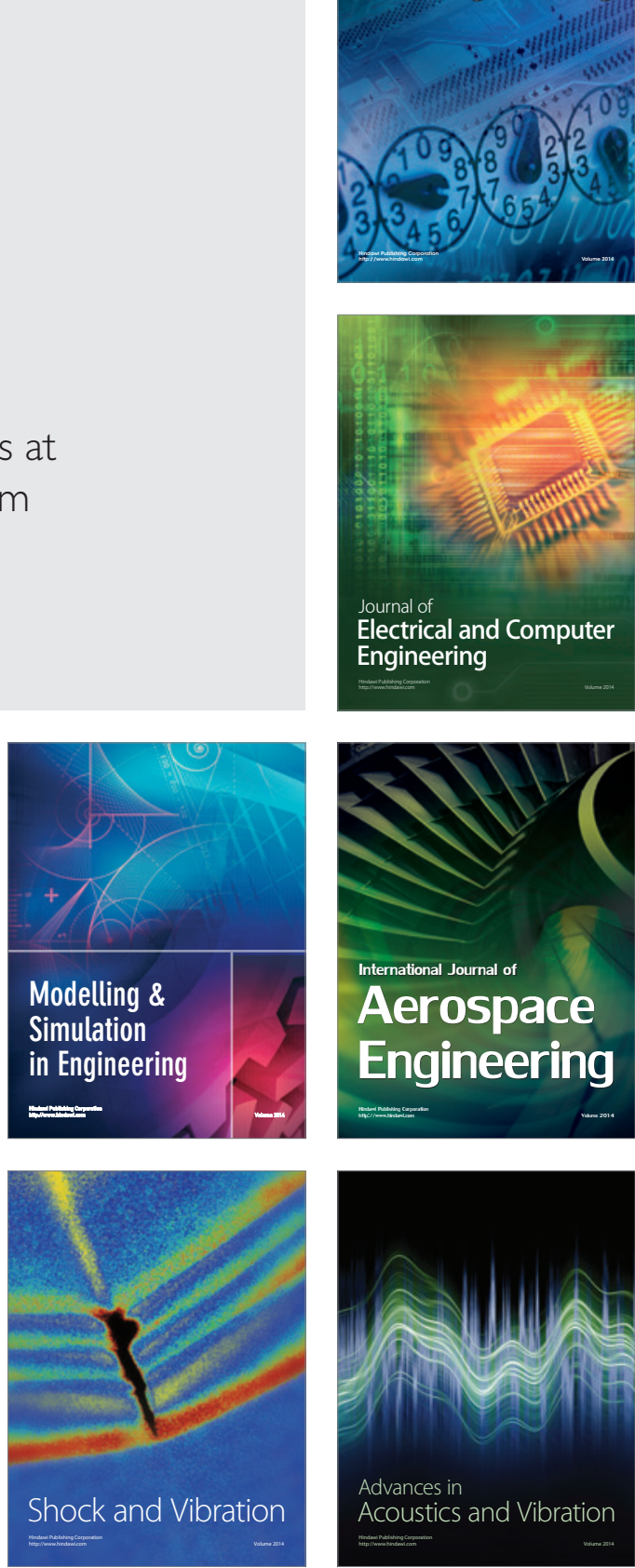